This is not the version of record. The full version of: Mohiuddin, Abdullah, Tarek, Taha, Zweiri, Yahya and Gan, Dongming (2020) A survey of single and multi-UAV aerial manipulation. Unmanned Systems, 8(2), pp. 119-147. is available at: https://doi.org/10.1142/S2301385020500089 


\title{
A survey of single and multi-UAV aerial manipulation
}

\author{
Abdullah Mohiuddin ${ }^{1}$, Taha Tarek ${ }^{2}$, Yahya Zweiri ${ }^{3,1}$, Dongming Gan ${ }^{1}$,
}

\begin{abstract}
Aerial manipulation has direct application prospects in environment, construction, forestry, agriculture, search, and rescue. It can be used to pick and place objects and hence can be used for transportation of goods. Aerial manipulation can be used to perform operations in environments inaccessible or unsafe for human workers. This paper is a survey of recent research in aerial manipulation. The aerial manipulation research has diverse aspects, which include the designing of aerial manipulation platforms, manipulators, grippers, the control of aerial platform and manipulators, the interaction of aerial manipulator with the environment, through forces and torque. In particular, the review paper presents the survey of the airborne platforms that can be used for aerial manipulation, including the new aerial platforms with aerial manipulation capability. We also classified, the aerial grippers and aerial manipulators based on their designs and characteristics. The recent contributions, regarding the control of the aerial manipulator platform, is also discussed. The environment interaction of aerial manipulators is also surveyed, which includes, different strategies, used for endeffectors interaction with the environment, application of force, application of torque and visual servoing. A recent and growing interest of researchers about the multi-UAV collaborative aerial manipulation was also noticed, and hence different strategies for collaborative aerial manipulation are also surveyed discussed and critically analyzed. Some key challenges regarding outdoor aerial manipulation and energy constraints in aerial manipulation are also discussed.
\end{abstract}

KEYWORDS Aerial platforms, Aerial manipulation, Aerial manipulators, Aerial grippers, Multi-UAV collaborative transport

\section{INTRODUCTION}

Aerial manipulation, in general, is referred to as an activity performed by aircrafts with hovering capability, for grasping, transporting, positioning, measuring by using an end effector or a gripper, attached to the aircraft [1]. Development of Unmanned Aerial Vehicles (UAVs) with manipulation capabilities has attracted high attention in recent years. UAVs with manipulators are useful for many applications such as remote inspection [2], [3], cutting high tension cables [4], packages delivery [5] and monitoring of hostile environments [6]. Aerial manipulators can perform tasks, where human access is limited such as; turning a valve [7] in an inaccessible location, performing the inspection on bridges [8]. UAVs with manipulators can also be useful

\footnotetext{
*This publication is based upon work supported by the Khalifa University of Science and Technology under Award No. RC1-2018-KUCARS.

${ }^{1}$ KUCARS, Khalifa University of Science and Technology, P.O. Box 127788, Abu Dhabi, United Arab Emirates. abdullah.mohiuddin@ku.ac.ae

2 Algorythma's Autonomous Aerial Lab, Abu Dhabi, UAE. tarek.tahadalgorythma.com

${ }^{3}$ Faculty of Science, Engineering and Computing, Kingston University London, London SW15 3DW, UK. y. zweiri@kingston.ac.uk
}

for canopy sampling [9]. Recently a UAV-manipulator was proposed to place a wrist band in the hands of a victim for search and rescue [10]. Researchers are also considering the collaboration of aerial manipulators with ground robots [11]. Aerial platforms are being considered for their potential ability to transport goods, quickly and cost-effectively. A recent study showed that drone delivery could help reduce the greenhouse gases [12]. Aerial platforms can also transport deformable linear objects such as hose, or ropes [13]. A demonstration of building a rope bridge using multi-rotors is shown in [14].

Aerial manipulation research benefits from the existing knowledge of manipulator designs and development of aerial platforms such as multi-rotors and helicopters. However, the design of manipulators and controllers for aerial platforms requires some special considerations which are typically not needed in ground-based manipulation and transportation. Specifically, these considerations include the stability of the aerial platform, since any motion of the manipulator or any interaction of the manipulator with the environment will directly affect the flight of aerial platform. Aerial platforms are also constrained to use low weight manipulators since any added weight increases the thrust requirements and the power consumption. All the above constraints require researchers to focus on multiple aspects of aerial manipulation.

\section{A. Motivation and scope}

The motivation for this paper is to benchmark the state of the art in aerial manipulation research. A review of multiple aspects of aerial manipulation would not only help professionals inside the field but also the professionals outside of the field to familiarize with state of the art in aerial manipulation and may also help them apply some of these methods to their specific application areas. The scope of this survey is based on the current active research areas in aerial manipulation which include, research in development of new aerial platforms for aerial manipulation, or improving the existing platforms, designing of aerial manipulators and grippers, improving the control of aerial platform and the manipulators, the interaction of the aerial manipulator with the environment and the collaborative aerial manipulation using multiple UAVs.

\section{B. Research groups involved in aerial manipulation}

The growing interest of researchers in aerial manipulation is also, evident from the increasing number of research groups involved in aerial manipulation. Some research groups and their areas of research in aerial manipulation and their active aerial manipulation projects are summarized in 
TABLE I

RESEARCH GROUPS WORKING IN AERIAL MANIPULATION RESEARCH

\begin{tabular}{|c|c|c|}
\hline RESEARCH GROUP & HARDWARE PLATFORMS & MAJOR RESEARCH AREAS/PROJECTS \\
\hline $\begin{array}{l}\text { Autonomous Systems, Control and Optimization Lab } \\
\text { Johns Hopkins University } \\
\text { https://asco.lcsr.jhu.edu/ }\end{array}$ & Quadrotors with manipulators & Aerial grasping \\
\hline $\begin{array}{l}\text { Autonomous Systems Lab } \\
\text { ETH Zurich } \\
\text { http://www.asl.ethz.ch/ }\end{array}$ & Quadrotors Helicopters & Aeroworks, AiRobots, Reely, myCopter \\
\hline $\begin{array}{l}\text { Aerial grasping and manipulation } \\
\text { Yale University } \\
\text { https://www.eng.yale.edu/grablab }\end{array}$ & $\begin{array}{l}\text { Helicoptor with gripper, } \\
\text { quadrotors }\end{array}$ & Design and control \\
\hline $\begin{array}{l}\text { Aerial Robotics } \\
\text { KU Leuven } \\
\text { https://www.mech.kuleuven.be }\end{array}$ & Innovative rotary platform & $\begin{array}{l}\text { Aerial platform development } \\
\text { Design and control, paylaod transportation } \\
\text { CargoCopter project }\end{array}$ \\
\hline $\begin{array}{l}\text { Drexel Autonomous Systems Lab } \\
\text { Drexel University } \\
\text { http://www.pages.drexel.edu/ bjr } 37 /\end{array}$ & Quadrotors 2-4 DOF dual-arm & Control \\
\hline $\begin{array}{l}\text { German Aerospace Center } \\
\text { https://www.dlr.de/ }\end{array}$ & Helicopter 7 DOF arm & Aerial manipulation \\
\hline $\begin{array}{l}\text { Hybridrobotics } \\
\text { Carnegie Mellon University } \\
\text { https://www.cmu.edu/me/hdr }\end{array}$ & Quadrotors & $\begin{array}{l}\text { Geometric control of dynamic } \\
\text { Aerial manipulation, multi-UAV aerial manipulation }\end{array}$ \\
\hline $\begin{array}{l}\text { Institute of Robotics and Intelligent Systems } \\
\text { ETH Zurich } \\
\text { http://www.iris.ethz.ch/ }\end{array}$ & $\begin{array}{l}\text { Helicoptor with gripper, } \\
\text { quadrotors }\end{array}$ & $\begin{array}{l}\text { Multi-UAV co-operative aerial } \\
\text { manipulator, multirotor design }\end{array}$ \\
\hline $\begin{array}{l}\text { Institute of Robotics and Mechatronics } \\
\text { http://www.dlr.de/ }\end{array}$ & Helicopter with arm manipulator & $\begin{array}{l}\text { Aerial manipulation for } \\
\text { inspection }\end{array}$ \\
\hline $\begin{array}{l}\text { Interactive \& Networked Robotics Laboratory } \\
\text { Seoul University } \\
\text { https://www.inrol.snu.ac.kr/ }\end{array}$ & SmQ platform,ODAR & Aerial platform development \\
\hline $\begin{array}{l}\text { Jouhou System Kougaku Laboratory (JSK) } \\
\text { University of Tokyo } \\
\text { http://www.jsk.t.u-tokyo.ac.jp }\end{array}$ & Transformable multi-rotor & Aerial platfrom development \\
\hline $\begin{array}{l}\text { MultiScale Robotics and Automation Lab } \\
\text { Purdue University } \\
\text { http://multiscalerobotics.org }\end{array}$ & & Aerial platfrom development \\
\hline $\begin{array}{l}\text { PRISMA Lab } \\
\text { University of Naples } \\
\text { http://prisma.dieti.unina.it }\end{array}$ & $\begin{array}{l}\text { Quadrotors with serial link } \\
\text { manipulators }\end{array}$ & $\begin{array}{l}\text { Development of aerial manipulation systems } \\
\text { for industry with ongoing project AeroArms } \\
\text { (www.aeroarms-project.eu) }\end{array}$ \\
\hline $\begin{array}{l}\text { Robotics Vision and Control Group } \\
\text { Universities of Seville } \\
\text { Pablo de Olavide University } \\
\text { https://grvc.us.es/ }\end{array}$ & Helicopters & $\begin{array}{l}\text { Aerial robotic manipulation } \\
\text { involving control, perception and planning } \\
\text { Current projects: Aerial Robot Coworker } \\
\text { AEROBI,ARCAS }\end{array}$ \\
\hline $\begin{array}{l}\text { Robotics and Mechantronics group } \\
\text { University of Twente } \\
\text { https://www.ram.ewi.utwente.nl }\end{array}$ & Aerial manipulator & $\begin{array}{l}\text { Control, ongoing project: Aeroworks, } \\
\text { AIROBOTS, SHERPA }\end{array}$ \\
\hline $\begin{array}{l}\text { SIRSLab - Siena Robotics and Systems Lab } \\
\text { University of Siena } \\
\text { http://sirslab.diism.unisi.it/ }\end{array}$ & Quadrotors & Aerial grasping \\
\hline $\begin{array}{l}\text { Robotics, Intelligent Systems \& Control (RISC) } \\
\text { KAUST } \\
\text { https://risc.kaust.edu.sa }\end{array}$ & Hexrotors, quadrotors & Passive aerial grasping \\
\hline $\begin{array}{l}\text { Vijay Kumar Lab (GRASP) } \\
\text { University of Pennsylvania } \\
\text { https://www.kumarrobotics.org }\end{array}$ & Micro-aerial vehicles & $\begin{array}{l}\text { Multi-UAV co-operative aerial } \\
\text { manipulation }\end{array}$ \\
\hline
\end{tabular}




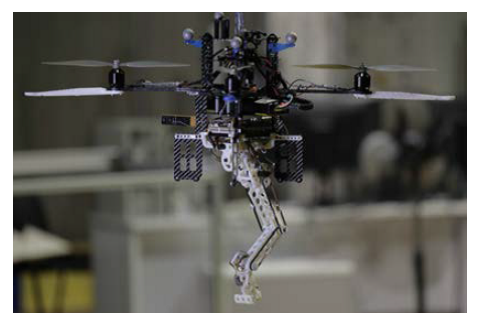

(a)

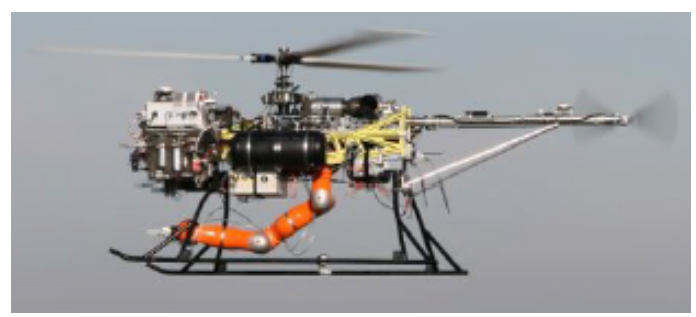

(b)

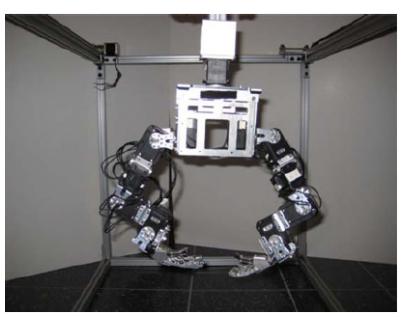

(c)

Fig. 1. Examples of a) multi-rotor with manipulator [15] b) helicopter with manipulator [16] c) simulation platform for aerial manipulation [17]

Table I. This list does not contain all the research groups currently active in the aerial manipulation but provides an idea to the reader about the people active in this field.

\section{Structure of the survey}

The paper is structured as follows: section II covers a brief discussion of aerial platforms, section III is about grippers and manipulators, section IV is presenting planning and control, section $\mathrm{V}$ is a survey of multi-UAV aerial manipulation, while section VI is regarding challenges of outdoor aerial manipulation and section VII is a review of the energy constraints of aerial manipulation. Some key observations and open research problems are discussed in the section VIII which is followed by the conclusion in section IX.

\section{Aerial Platforms}

Several aerial platforms with autonomous flight capability are currently available, such as multi-rotors, helicopters, fixed winged planes, but not all of them are well suited for aerial manipulation. Helicopters and multi-rotors have hovering ability which is not available with fixed winged planes. Some novel aerial platforms are also recently developed with hovering capability and promising prospects in aerial manipulation. Hovering capability allows an aerial platform to have a relatively fixed position in air and manipulator attached to the aerial platform can perform aerial manipulation tasks. A brief discussion of these aerial platforms which are used in aerial manipulation is provided in following subsections IIA II-B II-C II-D. The next subsection II-A will discuss multirotors, which are the most common aerial platform used in aerial manipulation research. Subsection II-B is about helicopters, and after that subsection II-C will introduce some novel aerial platforms with good potential for aerial manipulation. The last subsection II-D will present some simulation platforms that are used for aerial manipulation research.

\section{A. Multi-rotors}

Multi-rotors are most commonly discussed aerial platforms for aerial manipulation [18], [19], [20], [21]. The hovering capability present in multi-rotors provides an opportunity for the UAV to remain airborne while any manipulator attached to it, can perform a useful operation. Multi-rotors come in various configurations depending on the number of rotor arms, the orientation of rotor arms, number of propellers per arm, propeller configurations. Regarding the number of arms, the most popular are quad-rotors, hex-rotors, and oct-rotors. Based on rotor arm orientation, the most popular configuration is the cross configuration as compared to a plus configuration. The propeller configurations [22] can be either single propeller per arm or co-axial configuration, which means two propellers per arm. In single propeller per arm configuration, propellers can be placed above or below the arms. Multi-rotors are popular amongst the hobbyists and are commercially available; however, they have some constraints, when considering multi-rotors for aerial manipulation. Commonly used multi-rotors are underactuated systems, where translation motion depends on attitude control and any attempt in attaching a manipulator to aerial manipulator, must take the effect of manipulator on attitude control into account. In multi-rotors, the rotational direction of all rotors is kept such as to achieve a net zero reaction torque, which is obtained by rotating pairs of rotors in opposite directions canceling out each other's torque. The yaw motion is achieved by slowing down the opposite pairs of motors relative to the other pair. The yaw motion can be used to apply torque by attaching a gripper on top or below the multi-rotor. However, the amount of torque that can be applied is limited by the rotor capabilities and the length of the rotor arm. Another constraint with multi-rotors is the difficulty in scaling up. In simple terms, scaling up a multi-rotor with currently available technology is difficult, since the thrust produced by the rotors is increased by a square of the sweeping area of the rotors. Increasing the sweeping area of rotors requires the increase in the size of the quad-rotor which means the volume will increase. The weight or volume increases by the cube law. This simple analysis means that at some point scaling will not work. In general, rotor-crafts can be scaled up to a certain size [23]. It is also argued by [24] that scaling up a multirotor could also be dangerous and more expensive. Although, recent announcements of a large multi-rotor called Ehang is developed, till date no commercial or passenger flights are operated, or any data regarding the flight stability is provided. It is also important to note smaller quad-rotors are more agile [25]. The loss of agility while scaling up a multi-rotor might also result in less control or less ability to compensate for the inertia change due to the manipulator motion. Another constraint with multi-rotors as an aerial manipulator, is the reduced flight time, with a typical flight time of 15 to 30 
minutes [26]. The payload carrying capacity is also limited. Addition of a payload, also adversely affects the flight time. In general unilateral Electronic Speed Controllers (ESCs) are used for the motors of the multi-rotors. Multi-rotors with unilateral ESCs cannot exert downward force larger than their weight [27]. A manipulator can be attached to a multi-rotor as shown in Figure 1(a). Further discussion of design and control of multi-rotors with manipulators is discussed in sections III-B and IV-B. Recently [28] presented the design of fully actuated multi-rotor called as Tilt-Hex. Tilt-Hex provides better pose control. It can also apply force and torque independently with the help of a rigidly fixed manipulator [28]. As of today, no studies were found in the literature where a moving manipulator is attached to a TiltHex. This review will mainly focus on under-actuated multirotors and helicopters with manipulators.

\section{B. Helicopters}

Helicopters are also widely referred, in aerial manipulation literature after multi-rotors [29], [30], [31], [32], [16], [33], [34]. They also have hovering capability, like multi-rotors, but better payload capacity as compared to multi-rotors. Helicopters can be easily scaled up in size and payload capacity as compared to multi-rotors. Examples of helicopters with better payload capacity in the literature which enables them to fly with relatively heavy arm manipulator are [35], [16]. An example of a helicopter with an arm manipulator is shown in Figure 1(b). Unlike multi-rotors, a conventional helicopter consists of a single main rotor and a tail rotor. The main rotor is responsible for creating enough thrust for the helicopter to lift it. The translational motion of a helicopter is achieved by creating differential lift, by cyclic control of the angle of attack of the rotor blades. The ascending and descending motion can also be achieved by a collective change of angle of attack of both rotors, thereby changing the thrust produced. The rotation of single main rotor causes, a reaction torque, which is countered by the tail rotor. The yaw motion of a helicopter is achieved by increasing or decreasing the thrust produced by the tail rotor. The thrust produced by the tail rotor is adjusted by varying the angle of attack of the blades. The yaw motion generated by the tail rotor could result in a higher torque since the length of the tail adds to the yaw torque. This could be useful when a gripper is attached just below the helicopter and needs to perform an operation requiring high torque. Furthermore, the efficiency of a single but bigger rotor is higher than multiple small rotors. One big rotor can have more chances of creating a blowing effect, i.e., disturbing the object that is to be picked. One big rotor also means more chances of collision of blades while performing aerial manipulation so maybe a bigger manipulator is needed for any lateral manipulation. The main rotor also causes oscillation effects which makes a helicopter challenging to operate. Examples of these oscillation effects are mechanical resonance in air and ground resonance [36]. Furthermore, current helicopter models used in literature for aerial manipulation do not have any protective ring around the main rotor, which is sometimes present in multi-rotors and makes it safer for aerial manipulation. Blade flapping is expected to be higher in helicopters since the main rotor is longer hence more bending near the ends of the rotor. Aerodynamics of multirotor and helicopters are also different; it is obvious that a helicopter would be susceptible to more lateral drag because of the more lateral area. Hence wind disturbances will cause more positioning errors. It is not possible to attach a manipulator on top of helicopters, whereas it is possible and practiced in multi-rotor [37], [7], [38]. This also prohibits the use of helicopters for contact inspection under the roof like surfaces such as bridges. Helicopters are also generally less agile and have less maneuverability in tight spaces [39]; thus the addition of an arm manipulator would also impact its agility and maneuverability. Addition of arm manipulator also makes the system more sensitive to the vibration and resonance effects caused by the main rotor [36].

\section{Novel aerial platforms}

Most of the studies presented in the literature are using commercial UAVs for the aerial manipulation research. However, researchers have also proposed, novel platforms [47], [27], [44], modification to existing platforms [40], [48], [49], whereas [46] presented a technique of designing a new aerial platform based on application. Various new trends are being observed, one of them is exploring the possibility of hybrid aerial vehicles which can move on various mediums such as water and ground along with flight capabilities [42], [3], [49] and the other trend is creating re-configurable vertical take-off and landing aircrafts (VTOLs), which do not have fixed shapes [45], [44]. An account of all these is discussed below.

The modification was proposed by [40] in the form of the addition of a buoyant structure to the quad-rotor to overcome the instability of the flight for redundant manipulators as shown in Figure 2(a). Researchers planned to attached a manipulator to the proposed hybrid UAV. The test runs in [40] show that this hybrid structure without the manipulator does stabilize the flight. However having attached a buoyant structure such as the one shown in Figure 2(a) must add the aerodynamic effects such as parasitic drag. It also increases the lateral surface area which means that perturbations caused by wind might offset the benefit that is being achieved by the addition of buoyant structure.

Another novel aerial platform recently presented, is called, spherically-connected-multi-quadrotor platform (SmQ Platform) [47] as shown in Figure 2(b). Three quadrotors were attached to small beams using spherical joints. The small beams were concentrically connected to a small circular plate acting as the base frame. The connection of multiple quadrotors to a single frame allows increasing the total thrust force that can be generated by the aerial platform. SmQ platform is also able to execute yaw motion and can apply horizontal force using a link that is extending out from the base frame. The system although is over-actuated, but the actuation is limited by the range limit of spherical joints. Another novel aerial platform recently presented, is 


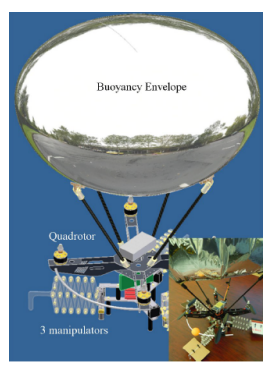

(a) Quadrotor with bouyant structure [40]

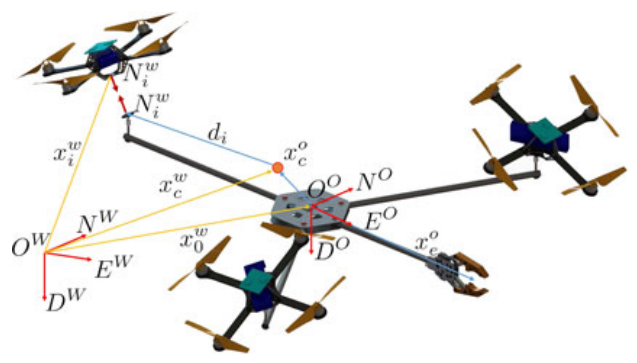

(b) Spherically connected multiQuadrotor [41]

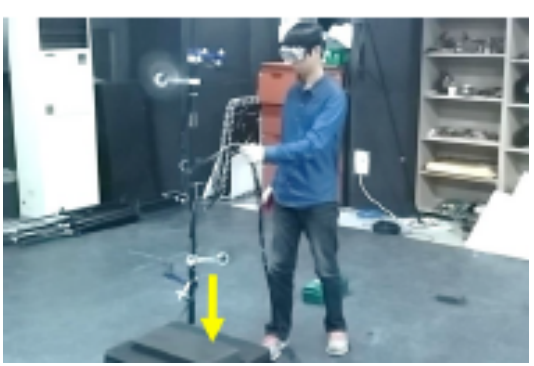

(c) Omni-Directional Aerial Robot [27]
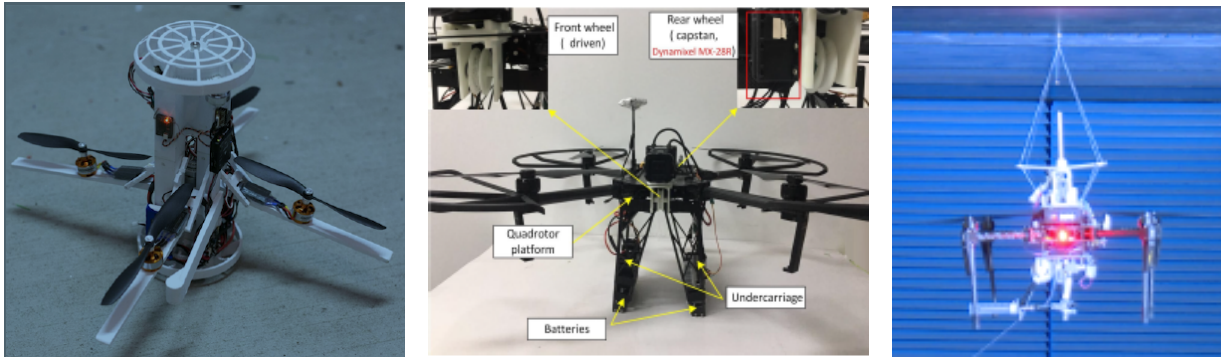

(d) Hybrid

ground

aerial (e) platform [42]

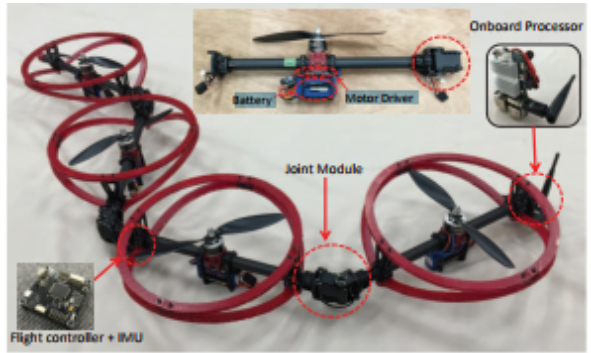

(g) Transformable aerial platform [44] of wires [3] Micro-Aerial Vehicle [43]

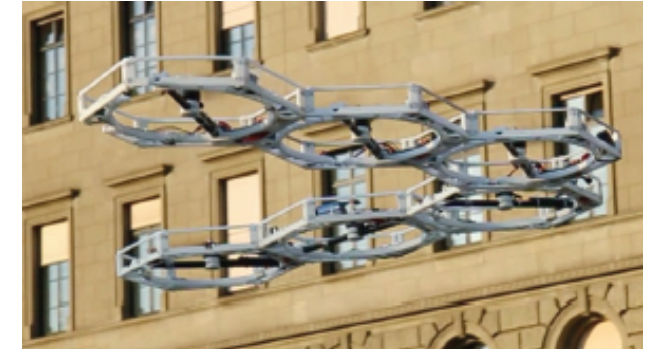

(h) A modular VTOL Vehicle [45]

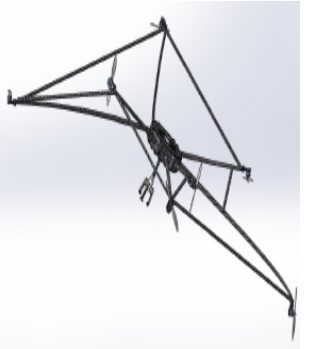

(i) Platform developed using the method presented in ref [46]

Fig. 2. Examples of novel aerial platforms discussed in literature

called, omni-directional-aerial-Robot (ODAR) as presented by in [27] as shown in Figure 2(c). ODAR is a fully actuated aerial platform with reversible ESCs which can produce a bidirectional current; hence the ODAR platform can apply force in both directions; a feature that is currently not available in conventional commercial multi-rotors and helicopters.

Researchers [42], [3], [49] have also tried to create a hybrid platform, which can not only fly but travel through another medium. One example [42] is shown in Figure 2(d) in which the aircraft can not only fly and hover in the air but also travel on the ground, after going through a transformation, where it folds its arms and forms a two-wheel vehicle. This kind of hybridization, can increase the reach of aerial platform further and also save energy by traveling through the ground when flying is not necessary. Another example is [49] where the aerial platform can not only hover and travel underwater, but it is also capable of flying in the air, during the same flight. In above two cases, the prospects of attaching a manipulator are yet to be explored, and integration of a manipulator to such platforms would require considerations, regarding the travel through another medium based on the requirements of the other medium. Another example [3] of an aerial platform traveling through another medium is shown in Figure 2(e), where the drone can hover in the air, but it can also land on wires for inspection, and after landing it can travel on wire, and fly again, in case of any obstacle on the wire. Another example [43] is the Figure 2(f), which has an additional mechanism to launch anchors. These anchors attach to the surroundings and hence provide support to the UAV. This anchoring or perching allows the UAV to save energy instead of the usual hovering in position performed during an aerial manipulation operation.

A novel concept is the use of a transformable aerial platform, where the whole platform can act as a gripper. In this case, the aerial platform consists of rotor arms, which can change their configuration, as compared to fixed rotor arm multi-rotors [44] as shown in Figure 2(g). In this type of arrangement, any external manipulator arm or gripper is not required. In a demonstration, it successfully transformed itself such that it encompassed the box, grasped it and lifted 
it. The transformation of the aircraft also makes it more successful in navigation through narrow passages as compared to other types of multi-rotors, since it can transform its shape based on the passage. Since the whole platform transforms and interacts with the object, it seems necessary to add a protective ring around the rotors to protect the rotors and the object. This is not necessary for conventional multi-rotormanipulator systems since a manipulator adds reachability and provides some disconnection from the instabilities of interaction with the object.

A similar concept is having a modular vertical and take off vehicle [45], as shown in Figure 2(h) where, multiple individual modules with single rotors, can join together via magnets to form various configurations. The individual modules use wheels to move around on the ground and connect to the other modules, and after forming a configuration, the modular VTOL can fly.

Another approach could be designing a platform from scratch, based on the application. Designing a new platform, instead of using existing ones, could provide more flexibility in terms of application-specific attributes that might not be present in existing quadrotors, hex-rotors or helicopters. These attributes could be a desired force or torque acting on the end-effector based on any application. Hence a technical analysis can be performed [46] to determine the mechanical structure of the system, the number of thrusts and geometry. A platform that was developed by following the method described in [46] is shown in Figure 2(i). It can be seen in the Figure 2(i), that the platform is quite unconventional in terms of its structure and the location of actuators on the platform.

\section{Simulated UAV platforms}

A platform that can mimic the aerial behavior of a UAV can provide a useful tool for the testing of heavy manipulators. Studies performed by [17], [40], [50], [51], [52], instead of using UAV, used a platform that can mimic the effects of flight. An example of such a platform is shown in Figure 1(c). The authors suggest that UAV simulating platform bridges the research gap between the current aerial platforms lacking high payload capabilities and thus the capacity for more dexterous heavy manipulators. A UAV simulator was developed by [40], [50], which uses a mathematical model of a quad-rotor to emulate the behavior. A six-degree-of-freedom miniature version of the gantry crane was used to replicate the UAV by [17], which provides the complete range of motion of a rotor-craft as well as ground truth information without the risk associated with free flight. These UAV test rigs, however, do not account for various aerodynamic effects experienced during highly dynamic flying maneuvers.

\section{AERIAL MANIPULATORS AND GRIPPERS}

Although the working principle of manipulators and grippers attached to a ground robot or an aerial robot are the same, however, the aerial manipulators and grippers require some considerations such as; aerial manipulators are designed while considering the effects of moving manipulator on the aerial platform. The grippers for aerial platforms are designed while considering the effects of positioning errors of the UAV during grasping of an object. The challenges imposed by aerial manipulation can be dealt with, via both improving the design and control of the systems; therefore researchers have attempted to improve not only the design of aerial manipulators and grippers but also the control strategies. This section is divided into two subsections, where section III-A surveys different types of aerial grippers and their characteristics and section III-B presents different aerial manipulators, some design examples from literature and characteristics of different types of aerial manipulators.

\section{A. Grippers as end-effectors in UAV-manipulator systems}

Aerial platforms can carry a wide range of low weight endeffectors such as NDT (Non-Destructive-Testing) sensors and grippers. NDT sensors require the design of manipulators and interaction control strategies to incorporate the effects of resulting instabilities during contact based inspection. Any design aspect of NDT sensors themselves does not seem relevant to aerial manipulation research hence the current section will focus on grippers attached to the aerial platforms. The most simple design of gripper for aerial manipulation is a hook [53], however it will require precise position tracking of manipulator and the aerial platform. Grippers are the most common type of end-effector. They usually have different gripping techniques and actuation styles. Grippers for aerial manipulators generally include the following characteristics: light-weight, payload shape compatibility, the reliability of grasping, the mechanism to deal with reaction forces, ability to compensate for positioning error of UAV. A comparison of different characteristics of common grippers used in aerial manipulation is provided in Table II.

1) Compliant grippers: A mechanically compliant system deals with position uncertainty of the UAV by incorporating the compliance in the gripper [54]. This ensures that the errors in position control of the UAV do not result in large forces, and the gripper conforms to the object. Grippers with compliance can be divided into two categories, active compliance, and passive mechanical compliance. Active compliance is based on active control, utilizing sensors and actuators which work together to get the desired, forcedeflection relation. Passive mechanical compliance, however, is achieved via attaching springs in robot joints, to create the allowance for large joint deflections. This results in lower contact forces.

An example of passive mechanical compliance is a single degree of freedom anthropomorphic finger module that was presented in [55] as shown in Figure 3(a) for aerial manipulation and grasping. The finger module was driven by a high torque motor that moves the three joints of the finger using a tendon. Elastic elements were used to keep the joints extended or open. This elastic element results in the passive mechanical compliance of the finger module against collisions with any objects. The authors extended their work in [56] by attaching the compliant finger to the compliant arm, which is further discussed in manipulator 
TABLE II

DIFFERENT GRIPPER TYPES AND THEIR ATTRIBUTES

\begin{tabular}{lllllll}
\hline Gripper types & Compliance & $\begin{array}{l}\text { Shape } \\
\text { compatibility }\end{array}$ & $\begin{array}{l}\text { Material } \\
\text { restriction }\end{array}$ & Manipulate & Grasp & Destructive \\
\hline Impactive gripper & Low & Low & None & Yes & Holds & No \\
Ingressive gripper & Low & Low & Yes (soft surface) & No & Penetration & Yes \\
Mechanical compliant gripper & High & High & None & Low & Holds & No \\
Magnetic gripper & None & Planar/curvature surface & Yes (Ferrous) & Low & Magnetism & No \\
Vacuum gripper & Low & Medium & None & Low & Self sealing suction & No \\
\hline
\end{tabular}

design section III-B.1. Another means of compliance in grippers can be achieved by adaptive under-actuation. A gripper with fewer actuators as compared to the degree of freedom shows adaptive behavior. One approach was from [54] as shown in Figure 3(c) which employs passive mechanical compliance via adaptive under actuation in a gripper to allow for large positional displacements between the aircraft and the target object. Another example of the under-actuated gripper is the study by [57] which discussed aspects of robot hand performance specific to grasping and perching from an aerial vehicle [57] also explored the impact of design and grasp parameters including tendon routing/pulley ratio, object size, and palm-size on the performance of both fully and under-actuated designs. It was shown that apart from perching applications, under-actuated designs consisting of single actuator per finger perform sufficiently, whereas for perching tasks fully actuated designs perform better.

Shape conformity is also achieved in a proposed aerial gripper [58], via using a prismatic joint to change the spacing of the fingers to match with the size of the object. After matching the size of the object the re-volute joints in the finger can rotate the finger links to wrap around the object.

An example of active mechanical compliance is the gripper which is a hybrid of an arm manipulator and gripper [59]. The concept is similar to dual arm manipulator with $2 \mathrm{DoF}$ fingers. The independent control of the fingers allows for more shape conformity.

2) Ingressive grippers: Ingressive grippers [60], [61] can grasp the objects without any well-defined attachment points. As shown in Figure 3(b) these grippers penetrate the metal hooks into the payload to attach themselves to the surfaces. That is why this gripper is useful for objects such as wood which can permit the penetration of hooks. Apart from that, the object must have enough planar surface area for the hooks of the gripper to penetrate. Ingressive grippers can cause some damage to the surface of the payload, which limits these types of grippers in applications which permit surface penetration of hooks into the payload. The advantage of this type of gripper is that it does not require to fully enclose the object to grasp it, whereas, the impactive grippers require the gripper to enclose the object for grasping as discussed below.

3) Impactive grippers: Impactive grippers [60] are those which use clamping motions to enclose the payload. Impactive grippers also grasp the object by applying sufficient normal forces or use frictional forces to hold the object; therefore it is necessary that the geometry of the payload be compatible with the gripper. It also requires creating enough friction between the contact surfaces of the object and the gripper. An example of the impactive gripper is [7], where an impactive gripper is attached on top of a hex-rotor as shown in Figure 3(d). The authors exploited the yaw motion of the hex-rotor platform, to create a torsional force that can accomplish tasks such as plucking the harvest, taking the light bulb off. Another example is in [62] where an impactive gripper grasped a foam brick.

4) Magnetic grippers: The gripping or grasping process can be simplified by using magnetic grippers for metallic payloads or payloads with metallic attachment points [63], [64], [24]. These magnetic grippers are commercially available and come in various specifications such as the one called OpenGrab developed by Nicadrone [65]. Some of these grippers use Electro-permanent-magnets which do not require a constant power consumption to stay activated. The magnets available in the market, however, do not take into account for any curvature of the payload surface, therefore in one study, the authors [63] designed a mechanically compliant structure that housed magnetic grippers, which enabled it to attach to surfaces with curvature successfully. Using a magnetic gripper and magnetic attachment points on payload simplifies some constraints associated with aerial gripping, on the other hand, it is also limited due to a magnet's load carrying capability.The biggest drawback of magnetic grippers, is their limitation to attach to ferromagnetic materials, which greatly restricts their application range. Magnetic grippers were also used in a recent Mohamed Bin Zaid International Robotics Challenge (MBZIRC 2017), where most of the teams used magnetic grippers to lift a metallic payload. The gripper developed by KAUST team, [66], [67] consisted of permanent magnets, and the object was detached by creating a gap between the permanent magnets and the object using a plate that is moved by a servo actuator. Magnetic grippers also accompany sensors such as infrared or camera to detect the object and to provide confirmation of attachment of payload.

5) Vacuum grippers: A vacuum gripper works by using suction created in the cup in contact with the payload surface [68]. Usually this is performed using a vacuum pump. The vacuum pump used in [68] comprised of $24 \%$ of the total mass of the quadrotor. The vacuum gripper in [68] showed the ability to successfully grasp a wide variety of objects including a USB plug, battery, wood block, eye glasses, hair 


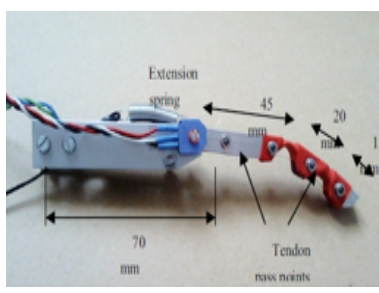

(a)

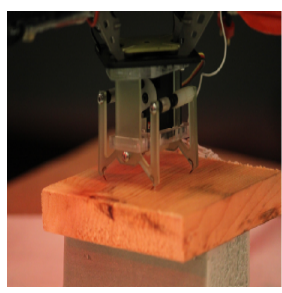

(b)

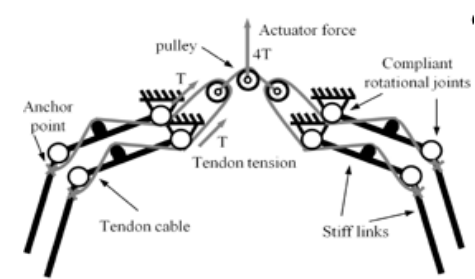

(c)

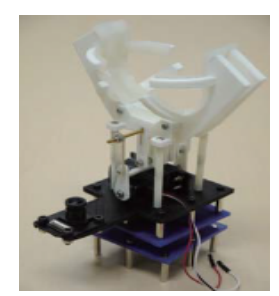

(d)

Fig. 3. Examples of grippers a) Compliant anthromorphic finger module [55] b) Ingressive gripper, gripping a wooden part [60], [61] c) Adaptive under-actuated compliant gripper [54] d) An impactive gripper for torque application [7]

brush, rubber duck, box, wood block, metal tin and plastic container with angled lid. The compliant behavior is added by using a spring-based universal joint supporting the plate with suction cups. However, the ability to conform to the shape of the payload is not available.

6) Electroadhesion based grippers: The principle of electroadhesion [69] is the electrostatic attraction. This type of attachment works with dirty or dusty surfaces, however the surface must be smooth. It requires, however, a continuous supply of small amount of energy to keep the attachment. Electroadhesion was used successfully as a perching mechanism for a micro-UAV by [70]. This method is promising for smaller UAVs. It is difficult to manufacture the grippers with actuators for small insect sized UAVs. The method is electrically and mechanically simple which is helpful in case of insect sized UAVs. The only requirement is the electro-adhesive patch and the voltage source. Another drawback of this method is the dependence of the method on environmental factors such as humidity, material properties, current leakage.

\section{B. Aerial manipulators}

A manipulator is defined as an object added to the multirotor to extend its reach or reduce disturbances to the airborne platform during interaction with the environment. Attachment of a manipulator to a multi-rotor changes the dynamic behavior of the multi-rotor due to, change of center of gravity $(\mathrm{CoG})$, the variation of inertia, and effects of dynamic reaction forces and torques generated by the movement of the arm [71]. One way to tackle this change of dynamic behavior is by designing appropriate control algorithms that can reduce flight instability, and the other way is to design manipulators that reduce these three causes of instability. Control methods to deal with these causes of instability are discussed in the control section IV-B, while this section is focused on the design of manipulators.

The design of manipulators attached to UAVs is strongly constrained by the weight of the arm, in fact, one of the most important design criteria is to improve payload to manipulator weight ratio. The weight of the manipulators can be reduced by the 1) selection of low weight material for manipulators 2) selecting low weight actuators. The designs of the aerial manipulators are influenced by the requirements of certain desired characteristics. These desired characteristics of the manipulators may include, ability to absorb the impacts, reach-ability, dexterity [72], and compliance. Certain types of manipulators exhibit different characteristics, which is why it is essential to discuss the types of aerial manipulators. Aerial manipulators can be classified into four basic types based on literature survey and the definition of manipulators as described at the beginning of this subsection III-B. Link-based serial manipulators, parallel linked mechanisms, hydraulic manipulators and cable manipulators. Each one of these types has different characteristics as summarized in Table III, and discussed below.

1) Link-based serial manipulators: Link-based serial manipulators are widely used in aerial manipulation as shown in Table IV which is a summary of contributions regarding serial link manipulators. Link-based serial aerial manipulators generally consist of rigid links, connected via joints which are actuated by servo motors. The complexity of serial link manipulators increases with the number of links. Usually, with every joint, a servo motor is required for actuation. Thus adding more links can increase inertia disturbances and cause offset in CoG. An approach in minimizing the disturbance caused by serial manipulators is by keeping the motor actuators close to the base of the arm [?], [76]. In one example of a serial manipulator, the first two DC motors are included into the base of the arm, while the last two are positioned at the beginning of the second joint. As shown in Figure 4(a) belts were used for the motion transmission for the third joint [?]. Keeping the motors close to the base constrains the center of gravity of the arm as close as possible to vehicle base, thus reducing the total inertia and static unbalancing of the system. The manipulator was able to fold on itself during landing, or when not in use. The CoG stays close to the vehicle base in folded position, which minimizes disturbances, especially during takeoff and landing. A deployable or foldable manipulator also helps in keeping the CoG disturbance to a minimum. In [76] the serial manipulator was based on tentacles based design for which actuators were close to the base, the motion was transmitted via cables. A deployable, low mass manipulator for UAV helicopter was developed by [29]. As shown in Figure 4(b), it was developed based on steel tubular booms which can be wrapped around a pulley and deployed when needed. Apart from the above approach, CoG change caused by the manipulator motion can be compensated by adjusting the battery position in the UAV. The battery is a heavy component of an aerial vehicle, and its position in the UAV 
TABLE III

CHARACTERISTICS OF DIFFERENT TYPES OF MANIPULATORS ATTACHED TO AERIAL PLATFORMS

\begin{tabular}{|c|c|c|c|c|c|c|c|c|}
\hline Manipulators & Dexterity & Reachability & $\begin{array}{l}\text { Reactionary } \\
\text { moments }\end{array}$ & $\begin{array}{l}\text { Inertial } \\
\text { change }\end{array}$ & $\begin{array}{l}\text { Change } \\
\text { of CoG }\end{array}$ & Weight & $\begin{array}{c}\text { Compliance } \\
\text { management }\end{array}$ & $\begin{array}{c}\text { Impact } \\
\text { on takeoff }\end{array}$ \\
\hline $\begin{array}{l}\text { Parallel linked } \\
\text { mechanisms }\end{array}$ & High & High & Low & Low & Low & $\begin{array}{l}\text { Number } \\
\text { of links }\end{array}$ & Elastic joints & Low \\
\hline $\begin{array}{l}\text { Linked based } \\
\text { serial }\end{array}$ & Higher & Higher & Higher & Higher & Higher & $\begin{array}{l}\text { Number } \\
\text { of links }\end{array}$ & Elastic joints & Higher \\
\hline Cable based & None & None & None & None & Low & Low & Good & Low \\
\hline $\begin{array}{l}\text { Hydraulic } \\
\text { manipulator }\end{array}$ & Low & Low & Low & Low & Low & High & None & Low \\
\hline
\end{tabular}

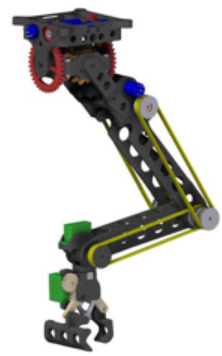

(a) Arm with motors close to base, and motion transmission with belts [15]

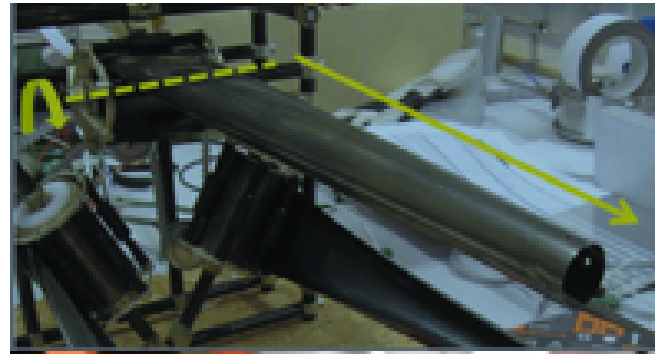

(b) A deployable manipulator [29]

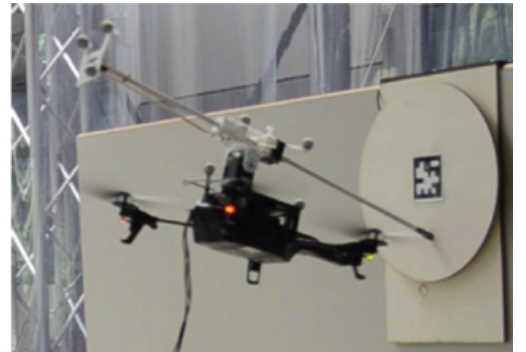

(c) Arm with interlocking mechanism for impact absorption [73]

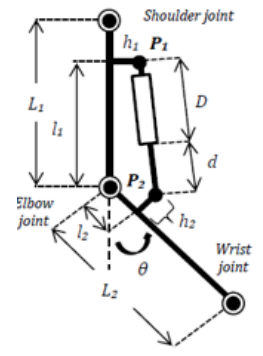

(d) Arm with linear servo and spring for compliance [74]

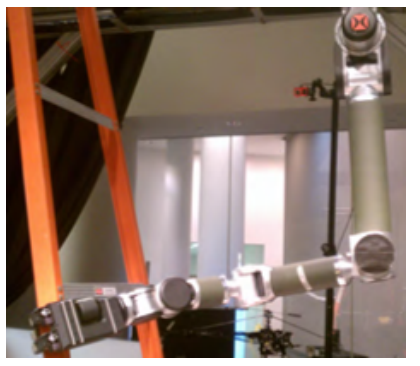

(e) A dexterous manipulator on a UAV simulator [50]

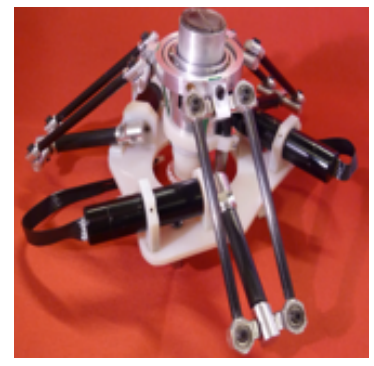

(f) $\mathrm{A}$
tor $[75]$

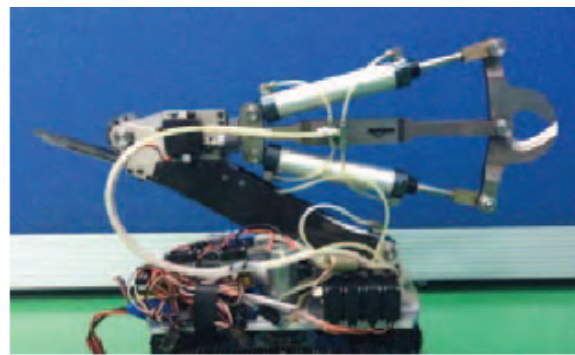

(g) A hydraulic manipulator [4]

Fig. 4. Examples of manipulators, including serial, parallel link mechanisms and a hydraulic manipulator

can affect the value of $\mathrm{CoG}$ of the aerial vehicle. A system was proposed by [21] which helped in balancing of the CoG by the short movements of the battery.

In general both serial and parallel linked manipulators are susceptible to unwanted force or impact that could result in the transfer of this force to the base of the UAV and hence cause instability. However, in this section, some examples of serial link manipulators are presented where researchers have attempted to reduce these effects. Any unwanted interaction is usually dealt with by incorporating passive mechanical compliance. Springs or elastic bands are used to achieve compliant behavior. As shown in Figure 4(c), [73] proposed the design of an aerial manipulator, which includes a passive joint capable of storing impact energy in the form of elastic potential energy. The impact compresses the elastic band, while decompression is prevented by using mechanical interlocking. The resulting manipulator can absorb the impacts without making the UAV-manipulator system bouncing away from the impact object. Elastic joints were also used by [56], but without the interlocking mechanism, however, in [56], elongation of spring in the joints is used to measure torque and deflection by using a potentiometer. Another example of elastic joints is [56] in which the joint was driven by a linear servo as shown in Figure 4(d). The linear servo and the link were connected via springs. These springs not only provided compliance but the elongation of springs also helps in determining the mass of the payload. Above methods of adding compliant behavior in manipulators are useful, but the compliant behavior, cannot be altered since it depends on the elasticity of the springs or elastic elements used. There is another method of ensuring compliance which is also reconfigurable. This method is based on impedance control of the end-effector, it is discussed further in the coming section IV-C.1, under the interaction control.

Serial link manipulators can be designed to have good reachability and dexterity. A hyper-redundant manipulator provides good reachability and dexterity and gives the flexibility to control the hovering position of the UAV [72]. It is 
also demonstrated that serial link manipulators can become hyper-redundant by adding more links. It enables the manipulator to use an extra degree of freedom to grasp objects which are usually not graspable with its end-effector. The extra degrees of freedom also help plan the manipulator tasks in a way that impacts on platform stability are minimized.On the other hand, having a higher degree of freedom and more links adds to the overall weight of the manipulator. Some aerial manipulators with good dexterity, including [72], [17], [50] were tested while they were attached to test-rigs or gantry. The paper [17] described the design of a system to emulate a flying, dexterous mobile manipulator. Two four degree-of-freedom manipulators were attached to the gantry system for performing the grasping tasks. Computer vision techniques and force feedback servoing provide target object and manipulator position feedback to the control hardware. Another study by [50] presented a design of a 7 degree of freedom arm with 4 degrees of freedom of the hand as shown in Figure 4(e), although the arm was attached to a test rig capable of mimicking an aerial platform, the authors claim that this manipulator can successfully manipulate and transport various objects while maintaining stable flight.

A novel type of serial link manipulators is a protocentric manipulator. A Protocentric manipulator as defined by [77] has multiple arms with first joints coinciding with the center of mass (COM) of the aerial platform. A protocentric manipulator can be used to grasp cylindrical objects with larger diameters by enclosing the object using at least two arms. It also seems possible to keep the inertial parameters similar by balancing the motion of one arm to the motion of another arm.

2) Parallel linked mechanisms: One way to minimize the disturbances caused by the aerial manipulators is to use delta manipulators [75], [87], [88], [19], [89] instead of a serial manipulator. Delta configuration of manipulators is usually very lightweight, and robust compared to a serial manipulator since the actuators are attached to the base [87], [53], [10]. If the delta manipulator is attached to the UAV such that the force acting on the delta manipulator is passing through the $\mathrm{CoG}$ of the manipulator, it can counter some moments acting on the aerial platform due to the reactionary forces of the manipulator. Another example of delta manipulators is demonstrated in [75] as shown in Figure 4(f), where authors developed a non-destructive testing end-effector on a three degree of freedom delta robotic manipulator. The sensor is mounted on Cardan gimbal joint that allows the sensor to interact compliantly with the remote environment. Another delta parallel links manipulator was designed by [88]. The manipulator was attached at the base of the UAV and is capable of moving its end effector in the regions below the UAV and on its sides. The authors used a parameter "global conditioning index" to come up with the design values for the length of the links. Global conditioning index gives a quantitative measure of the dexterity of the arm. The proposed manipulator is actuated by motors in pitch and planar directions. This is the only example of a delta manipulator with good reachability, while other delta manipulators are usually smaller and are attached on the side of the UAV along with a sensor and UAV has to move to perform the task. A delta manipulator causes fewer perturbations to the host platform, which makes it better than a serial manipulator, but usually, delta manipulators are smaller with less reachability; hence the improvement of reachability for delta manipulator as proposed by [88] proves that delta manipulators with good reachability can be designed.

3) Hydraulic manipulators: A low weight manipulator is favorable for aerial platforms, but in the case of a high torque requirement from an aerial manipulator, the criteria would be weight to power ratio. Applications such as cutting high tension cables require a very powerful end-effector which could provide very high power in a very short time. If electric motor actuators will be used higher torque can be achieved by the use of gears but that will also add additional weight and disturbance from the gearbox. A hydraulic manipulator as shown in Figure $4(\mathrm{~g})$ can be used to achieve such power. Although a hydraulic manipulator can be serial or parallel linked, it is classified differently here because of the actuation method and the promise of high torque. A hydraulic manipulator was proposed by [4] to attain high force/torque ratio. A hydraulic manipulator is well suited for high payload capacity aerial systems since a hydraulic system is heavy. However, it is advantageous over electric systems, since it is actuated by the flow of oil, which results in a smooth motion, i.e., fewer oscillations for host platform, and for the same power output, its inertia is less than other systems. The proposed hydraulic manipulator was not experimentally tested, so a physical demonstration of the workability of the proposed system is yet needed.

4) Cable based manipulators: Cable-based manipulators are easy to implement, however with cable manipulators, force can be applied by the pulling motion of the UAV only. Cable-based manipulators are compliant in nature; however, there is no dexterity. A simple cable based manipulator lacks the actuators, that can move the end-effector. Therefore, UAVs with cables, need to reach the target grasping position accurately, hence, precise positioning of UAV will be required to be able to grasp an object. In one example [64] the object was attached to the UAV using a string, and a magnetic gripper. A string provides more compliance as compared to rigid links, regarding the interaction forces between the payload and the aerial platform, however, during the grasping process, it is also susceptible to oscillations, hindering the attachment of the payload to the gripper. A Stewart-Gough inspired cable based magnetic gripper can provide compliance in the vertical axis and reduced oscillations in the lateral axis. The compliant nature of cablebased manipulators is very useful when multiple UAVs are used to lift an object [90], [5], [91]. This allows the UAVs to space out in the air, preventing chances of collisions. The orientation of the payload, cannot be altered by a single cable UAV system, however, in case of multiple UAVs carrying an object, it is possible to change the orientation of the payload by changing the relative configuration of the UAVs [90], [5], [91]. 
TABLE IV

SERIAL MANIPULATORS PRESENTED IN LITERATURE

\begin{tabular}{|c|c|c|c|c|c|}
\hline Ref & DoF & Purpose & Environment & Aerial platform & Year \\
\hline$[78]$ & $1 \mathrm{DoF}$ & Avian inspired grasping & Indoors via Vicon Motion sensors & Quadrotor & 2013 \\
\hline$[72]$ & $9 \mathrm{DoF}$ & Hyper-redundant aerial manipulator & Indoors & Test gantry & 2013 \\
\hline [79] & $2 \mathrm{DoF}$ & Controller design & Indoors via Vicon Motion sensors & Quadrotor & 2013 \\
\hline$[36],[16],[35]$ & $7 \mathrm{DoF}$ & Grasping via redundant DoFs & $\begin{array}{l}\text { Outdoors GPS navigation, } \\
\text { vision for manipulation }\end{array}$ & Helicopter & 2013-14 \\
\hline$[18]$ & Dual 2 DoF arm & Valve turning & Indoors but via visual servoing & Quadrotor & 2014 \\
\hline$[80]$ & $2 \mathrm{DoF}$ & Grasping using model pridictive control & Indoors via Vicon Motion sensors & Quadrotor & 2015 \\
\hline$[?]$ & $5 \mathrm{DoF}$ & $\begin{array}{l}\text { Reduction of disturbance by } \\
\text { keeping actuators close to base }\end{array}$ & $\mathrm{NA}$ & Quadrotor (intended) & 2015 \\
\hline$[38]$ & $3 \mathrm{DoF}$ & Inspection by contact & Simulation and outdoor experiement & Octrotor & 2015 \\
\hline$[21]$ & $6 \mathrm{DoF}$ & $\begin{array}{l}\text { Battery motion to counteract } \\
\text { manipulator disturbance }\end{array}$ & Indoors via Vicon Motion sensors & Octrotor & 2015 \\
\hline$[81],[82],[83]$ & $3 \mathrm{DoF}$ & Open a drawer, grasp a cylinder & Indoors vision and vicon & Quadrotor/hexrotor & 2015-16 \\
\hline$[56],[74],[84]$ & $\begin{array}{l}3 \mathrm{DoF} \\
\text { single/dual arm }\end{array}$ & $\begin{array}{l}\text { Compliant interaction and } \\
\text { minimizing disturbance }\end{array}$ & $\begin{array}{l}\text { Indoor/outdoor experiment } \\
\text { with visual servoing }\end{array}$ & Fixed base/hexrotor & $2015-17$ \\
\hline$[85]$ & $3 \mathrm{DoF}$ & $\begin{array}{l}\text { Self CoG adjusting arm design, cancellation } \\
\text { of torque produced by arm actuators. }\end{array}$ & Indoor and outdoor GPS & Hexrotor & 2017 \\
\hline$[86]$ & $1 \mathrm{DoF}$ & Force application $(20 \mathrm{~N})$ & Outdoors & Octrotor & 2017 \\
\hline
\end{tabular}

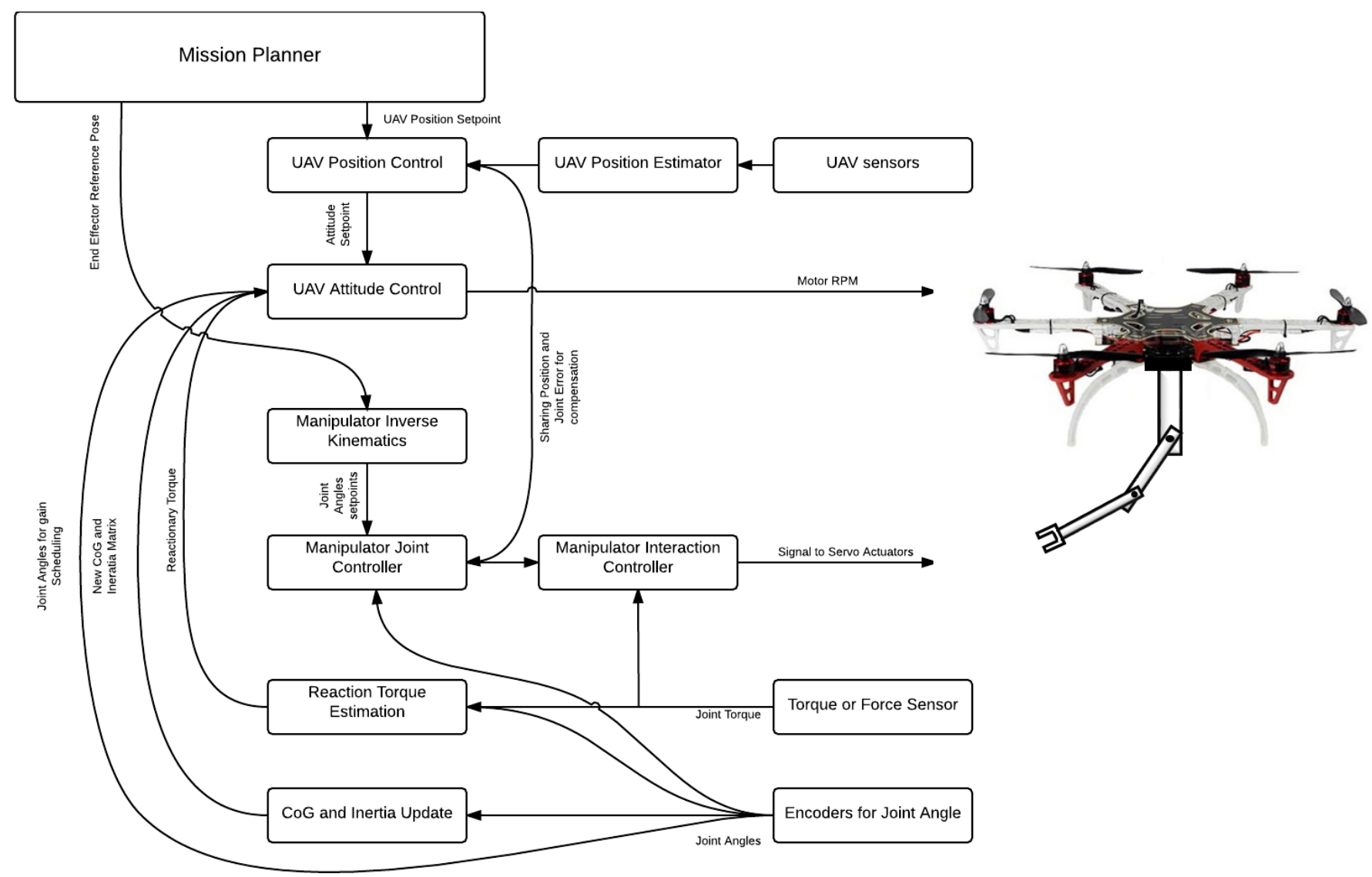

Fig. 5. A summary of different methods adopted in decoupled control scheme of UAV and manipulator 


\section{Planning And control of an Aerial MANIPULATOR}

Previous sections II and III covered the literature review regarding the hardware used in aerial manipulation. Current section will focus on the high-level planning, control, and environment interaction of the UAVs in subsections IV-A, IV-B and IV-C respectively.

\section{A. High level planning}

High-level planning is crucial in the cases where multiple UAVs have to work for a shared goal cooperatively. A highlevel planner in these cases plans the mission and then commands the low-level controller of each UAV. A similar scenario was presented in [63], [66], [92] where high-level planning was required to ensure optimization, obstacle avoidance, and co-operation between multiple UAVs. Specifically, the UAVs were required to autonomously, navigate in the shared area, detect the colored objects, grasp them and move towards the drop zone to drop them. Similarly, if the object is moving, it is crucial to detect the object and its motion, and then trajectory planning is required for gripping the moving object [93]. After grasping the object, planning is required to track the trajectory of grasped payload [94].

Before planning an operation such as grasping an object via UAVs, it would be useful to extract some information about the objects in the environment. This information could be the shape and pose or orientation. Object extraction is basically a concept where UAVs can get the information about the payload or the object which is supposed to be lifted or manipulated. Object extraction could be very useful where the mission control does not have prior information regarding the shape and pose or orientation of the payload. This is very critical to aerial manipulation since grippers require the compatibility with the shape of the object. Object extraction relieves the necessity of prior access to the model of the payload. An onboard object extraction method was developed by [95] that calculates information necessary for autonomous grasping of objects. In [96], a CNN (Convolution neural network) module was used to detect the tree branch where the UAV can perch. In [97] a Field Programmable Gate Arrays (FPGA) implementable vision-based orientation control system for an aerial robot is developed, which allows the multi-rotor to perch on bar like objects. A monocular camera was used in [97] along with an FPGA. The algorithm which can be implemented in FPGA is useful specially when high performing GPUs are not attached or available to the UAV. After getting information about the object to be manipulated via object extraction methods or having prior information about the object, the UAV-manipulator system can interact with the object.

\section{B. Aerial manipulator system control}

The control of a UAV-manipulator system brings forth challenges such as balancing of ground force effects, forces by the load, hover precision, flight stability, aerodynamic disturbances and the effects of manipulator motion. The motion of manipulator results in the change of inertial parameters. Addition of multiple links, joints, and actuators can increase the DoF, but it comes with a price, i.e., increase in the disturbances related to inertia. In case of multilink serial manipulators, the aerial platform's control may require a real-time update of inertial parameters as per the movement of the arm joints. Another constraint faced by the UAVs during aerial manipulation, especially in autonomous load transport is the disruption to flight stability by the instantaneous addition of a payload or addition of a payload with offset causing bias forces. Any torque that acts on the manipulator's joints, from environment interaction, is transferred to aerial platforms. A mechanism to deal with such reactionary moments is also required. The following subsections will discuss the strategies presented in the literature which provide solutions to the above-mentioned problems. The control strategies for an aerial manipulator system can be divided into two methods. One of the approaches is to have a centralized controller for both the UAV and the manipulator, and the other method is a decentralized controller.

1) Centralized control: A centralized controller, for a UAV-manipulator system, considers the complete dynamic model of the UAV-manipulator system, and it controls both the aerial platform and the manipulator. One of the centralized control was developed by [30]. A combined model was developed for the helicopter-manipulator system, and then a trim point based on manipulator mass was used to linearize the system. A linear quadratic regulator was developed and tuned to control the system. This approach has its limitations since it requires the knowledge of trim points based on the manipulator mass. The performance of the controller developed by [30] was acceptable in the steady-state region, and it is not expected to perform well in disturbances and away from the trim points. The authors, therefore, proposed the development of a centralized nonlinear control scheme for UAV-manipulator system. Till date, a centralized non-linear controller is not available in the literature. Furthermore, the controller developed by [30] was tested in simulation; hence a hardware experiment is needed to verify the performance.

2) Decentralized control: A decentralized control strategy involves two separate controllers for UAV and manipulator system. In this type of control strategy, the manipulator motion is considered a disturbance to the control of the aerial platform. The control of an under-actuated multirotor, with or without a manipulator, requires an outer loop position control which contains an inner loop attitude control. When a manipulator is added to the multi-rotor, the attitude control has to compensate for the manipulator motion or any reactionary torques. The following subsections are about the inner-loop attitude and outer loop position controllers for multi-rotors with manipulators.

Attitude control of UAV-manipulator: A decentralized control strategy requires some special considerations in terms of the stability of the whole system. A moving manipulator causes some instability in the flight of the UAV. This can be compensated by updating the inertia and CoG of the aerial manipulator system in real time. As shown in Figure 5, it is 
useful for the attitude controller to get a real-time update of the reactionary torque acting on the manipulator [98], [99], [100], [101]. The reactionary torque can be obtained by placing a force sensor on the base of the arm manipulator, or it can be calculated by using the joint angles if the force sensor is attached to the end-effector. Another approach to deal with the instability caused by moving manipulator is to use gain scheduling, [71], [98] where the gain depends on the realtime update of the joint angles of the manipulator. In other approach manipulator's joint angles can be identified which can provide the least disturbance during flight [62] for simple PID controller. Researchers [102], [16], [103], [104] have suggested, that traditional PID control for the attitude loop is not sufficient for having a smooth performance. An outer loop based on Model reference adaptive control (MRAC) was proposed in [102] to remove the oscillations that are not prevented by PID control loop only. Other controllers proposed for attitude control are Direct fuzzy logic [105], Adaptive fuzzy logic controller [105], Quaternion-based backstepping approach [103], IDA PBC [104], Variable parameter integral backstepping controller [106], feedback linearization approach and finite-time control of the double integrator system [99]. Quaternion-based backstepping approach [103] was able to compensate for the changes caused by movement of the arm. Variable parameter integral backstepping controller [106] was able to overcome the oscillations that were happening while using PID attitude controller. The double integrator system [99] was able to reject the parametric uncertainties and external disturbances. The intelligent controllers [105] were able to provide good trajectory tracking with the payload attached. IDA PBC [104] provided bounded trajectories for the bounded motion of the manipulator. In all the above examples goal was to improve the attitude control of a UAV with a manipulator, however, in one study [107] researchers proposed that it is possible to exploit the motion of manipulator to intentionally change the CoG of the UAV-manipulator system, so that the limits of maximum attitude angle can be increased.

Position control of UAV-manipulator: The constraints of imperfect position control of UAV-manipulator, during aerial manipulation, can be addressed by sharing the UAV's position or position error information with the manipulator controller, so that manipulator controller can compensate for the UAVs position errors by moving the manipulator accordingly [106], [103], [34]. Similarly, end-effector's position or position error can be shared with the position controller of the UAV, so that position controller can compensate for it, by moving the vehicle accordingly [106], [103]. This strategy is helpful, in cases, where decentralized control of UAV and manipulator is achieved by considering the movement of the manipulator as perturbations to the host platform. One approach is to use adaptive outer-loop horizontal position and altitude control [108]. The adaptive altitude controller in [108] generates the required thrust to hover, after considering the error in altitude, while adapting to any change in inertia and mass due to the motion of the manipulator. Similarly, the adaptive horizontal position controller, gen- erates the attitude commands after considering the error in horizontal position, while adapting to any change of inertia and mass due to manipulator motion. The resulting adaptive position controller was able to stabilize the platform, from the disturbances caused by the movement of the manipulator.

Aerial manipulation, especially, for near ground operations such as picking up an object, would be prone to the disturbances caused by aerodynamic forces arising due to proximity to the ground [109]. The solution proposed by [109] was to quickly descend after spotting the object and lifting it up. One of the solutions for this limitation is to let the UAV hover above the object to let the UAV settle, and when the error in position is at its minimum, the next setpoint for the position can be sent to start the descent towards the object.

\section{Environment interaction}

The control of the aerial platform with a manipulator was discussed in the previous section IV-B, which allows the UAV-manipulator system to be able to move in the air while staying stable. However, an important requirement for the usefulness of a UAV-manipulator system is its ability to interact with the environment. This interaction could be performed via, different approaches as shown in Figure 6. The following sections will discuss the impedance control, force control, torque control and object interaction via visual servoing.

1) End-Effector interaction control: The ability of the UAV-multi-DoF-manipulator system to have a stable hover with moving manipulator, i.e., varying joint angles is crucial for aerial manipulation. Moreover, another advanced aspect of UAV-manipulation is its end-effector's stable interaction with the environment. The stable interaction of the manipulator's end-effector can be achieved by making it compliant to external forces. It was discussed in section III-B.1 that compliant behavior can be achieved by using elastic elements, but elastic elements have fixed behavior, however, if compliance is achieved by using impedance control, the elastic and damping, behavior of the system can be configured based on the requirements [50], [110], [111], [112], [113], [71], [114], [115]. The impedance control can be classified based on the final control signal, which could be the joint angles of the arms or position based impedance control, or torque acting on arm joints. Apart from this basic classification, the sequence based interaction control structure is described in Figure 7.

1.1) Position based interaction control: The preference of position based interaction control over torque based interaction control comes from the fact that [71], although some larger servo motors may have the capability to control torque, the choice of joint angle based admittance control is due to poor accuracy of torque sensors. It is shown in Figure 7 that position based impedance controller is implemented in literature consisting of multiple layers [112], [111], [50], whereas a position based admittance controller is implemented in [71]. In case of [112], [111], [50], the endeffector's reference trajectory is provided to the impedance 


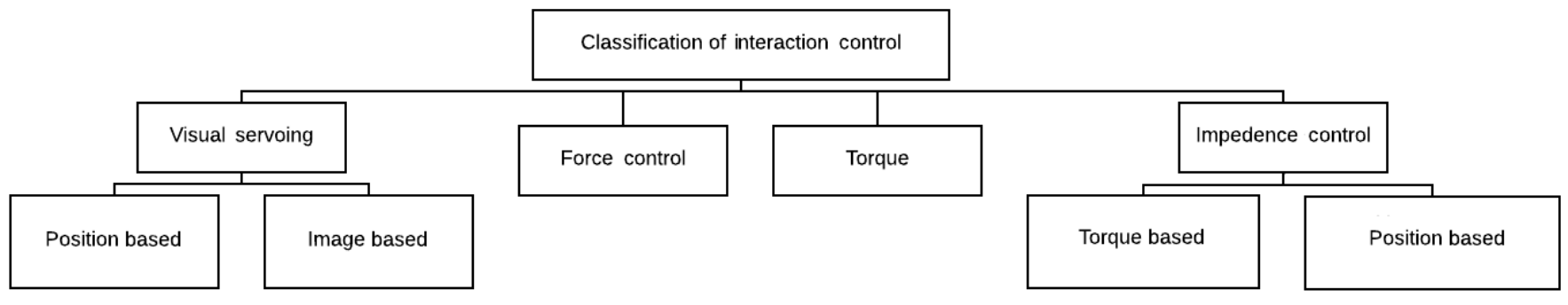

Fig. 6. A single UAV-manipulator system's interaction control hierarchy

controller, while in case of [116] joint angles are provided to the impedance controller. The required joint angles are sent to the joint controller in the end for position based impedance controller.

Impedance control [50] takes the required or desired trajectory and based on force sensor input data and the current trajectory, the impedance controller refines the trajectory, to reduce the effects of reactionary forces. This new trajectory is then implemented by a motion controller. An admittance controller was proposed by [71], which calculates the desired position of the end effector, as the sum of the distance from the desired position and the distance that is required to exert the necessary force. Another similar approach was presented by [112] in which controller was based on an impedance outer layer, which generates a compliant trajectory that results in bounded forces of interaction with the environment. The inner loop uses inverse kinematics to generate joint commands, while the innermost loop tracks the motion.

In case of [116], a motion planner, on the basis of the desired trajectory for the end-effector, determines the desired motion for the actuated variables (joint positions, quadrotor position, and yaw angle). The second layer utilizes an impedance filter to provide a selective compliant behavior to the system, for the interaction of the manipulator end-effector with the environment. Finally, the third layer implements a motion controller aimed at tracking the references output by the previous layer.

The multi-UAV grasp of an object with multi-link serial manipulators presents a challenge for control of the interaction forces between UAV-manipulators and the jointlyheld object. The object or payload, if grasped by two UAV-manipulators can have any unbounded force flow from one UAV-manipulator to the other UAV-manipulator. This unbounded force flow can de-stabilize the flight. The unbounded force flow can be bounded by an impedance controller as proposed by [111]. First impedance layer generates the reference trajectory to limit the external forces while the second filter deals with the internal forces between the manipulator and the UAV. The trajectory generated by the two filters are then used by a motion controller which uses inverse kinematics and a PD controller to implement those reference trajectories. Another approach for aerial manipulation with multi-link arms is discussed by [110], where a three-layer control architecture is proposed. The first layer is a centralized layer that generates trajectories for all end- effectors. The second layer is local to all UAV-manipulators, which computes motion references to track such end-effector trajectories coming from the centralized upper layer. The third layer is a low-level motion controller that tracks these motion references. It seems that a combination of approaches by [111], [110] would be better than using them individually, as explained below. Addition of the high-level top layer of [111] to the control strategy of [110], would make the process even safer and smoother. Both [111], [110] are about multi-UAV manipulation, but in [111] the internal forces between manipulator and UAV are also accounted, and in [110] a high-level layer takes into consideration of all end-effector poses.

1.2) Torque based impedance control: A different approach is torque based impedance control proposed by [113], in which the control input is the desired trajectory and current trajectory, and the control signal is the torque commands sent to the arm joints. Implementation of impedance control using torque would require taking care of sensor inaccuracies. The controller should be made [113] robust against any bounded force sensor inaccuracies and bounded unstructured modeling (non-parametric) uncertainties and/or disturbances in the system. In [117] similar strategy was presented, but an adaptive controller was proposed that estimates the dynamic model parameters.

2) Contact force control: Applications that require contact between an aerial manipulator's end effector and the wall would require the aerial platform to be able to control these forces. This type of force control is effective in contact based sensing applications, where a smaller delta manipulator with a sensor is attached on the side of a UAV. It was shown by [19] that when a UAV with a delta manipulator on its side, comes in contact with a wall, due to the reference position being virtually inside the wall, the actuators of the multirotor, will exert a force similar to a spring mass damper system. The force exerted during contact with the wall can be regulated by careful selection of the lateral reference position of UAV and manipulator set-points. A PI force controller was implemented by [89] on the manipulator in the direction normal to contact to regulate the force applied. A UAV-manipulator system was also developed by [118] for application of force, in which after establishing contact, desired attitude angles are calculated based on the required force; however, there was no feedback involved regarding the estimation of force or sensing of force for its regulation. 
In [86] a 1 DoF arm was used to achieve a force of $20 \mathrm{~N}$ for the hammering inspection of old walls. In this study, the force sensor was used, and force control was integrated with position control. The mean error was around $4.27 \mathrm{~N}$ which might suit the hammering test but not suitable for other applications where more accurate force control will be required.

3) Applying torque control: Yaw motion of a multi-rotor can be used to apply torque [7]. The yaw motion of a multirotor is caused by the difference in the angular speeds of the counter and clockwise rotations of the propellers. The torque generated by the multi-rotor is theoretically the sum of reaction torque produced by each propeller of the multirotor. An impactive gripper as shown in Figure 3(d), just attached below a multi-rotor was used in [7] to demonstrate unscrewing a light bulb. An increased amount of torque can be applied, via using dual arms attached to a multirotor [18], by increasing the moment arm between the forces. The torque applied can be increased further, if torque is applied by multiple UAVs. A similar concept was presented in a newly developed SmQ platform by [47], where multiple quadrotors were attached to a single frame, and a yaw motion was demonstrated. This concept can be exploited to achieve a high torque and would be useful in applications where high torque is necessary. It is also possible to not only control the torque by increasing and decreasing the force, but also by changing the point of application of the force. The point of application of force can be changed by simply attaching the multiple UAVs, closer or farther on the object.

4) Environment interaction via visual servoing: All the above control strategies involve the position based or torque based control methods, but recent studies by [82], [119], [120], [51], [121], [20], [64], [35], [122] utilized visual servoing to perform aerial manipulation tasks. Visual servoing requires attachment of the camera, which adds a little more mass to the UAV, and needs a bit of extra power to use the camera. Visual servoing is also very dependent on lighting conditions, and it won't work with insufficient lighting. This section describes different uses of visual servoing technique in aerial manipulation. The tasks including navigating towards payload, directing the host platform towards the desired location, maintaining a ready pose of the manipulator, picking up the object, transporting the carried object towards the target location, are performed in studies described below using visual servoing. Apart from visually servoing the aerial manipulator system towards the payload, [64] showed vision based search, docking, and lifting of the payload. The authors used visual servoing to approach and attach the magnetic end-effector to the correct spots and then lift the object up. The next subsection IV-C.4.1 describes the types of visual servoing methods.

4.1) Types of visual servoing: Visual servoing can be done in two ways, position based visual servoing [120] and Imagebased visual servoing (IBVS) [51], [20]. In position based visual servoing, markers are used to estimate the position, and then a control strategy is developed based on position estimation, however, an IBVS is based on control law, which is based on the error between desired and current features on the image plane, and does not require any estimate of the position. Position based visual servoing is least susceptible to lead the marker outside of the field of view, whereas image-based visual servo (IBVS) is usually done by velocity commands and there is always a chance of losing the sight of the marker. Researchers suggested using fish eye [82] camera to enhance the field of view for the case of imagebased visual servo, which then requires to do some image processing to convert it into perspective camera image. During roll and pitch the platform tilts; therefore the image is distorted, an image adjustment method is developed to address this issue. The control strategy starts with the raw image, which is used to calculate blob image. The blob image is converted from fish eye to perspective camera image. After extracting contour, the compensation of roll and pitch are performed, and then moments are calculated from the image to be used to calculate the velocity commands [82]. Authors including [119], [82], [51], [20], [64] developed their control strategies based solely on IBVS whereas [120] combined both image-based visual servoing and position based visual servoing by means of selecting one of the above methods based on the task. The tasks are defined as first moving towards the object and maintaining the desired position, which is achieved by position based visual servoing with the help of a downward facing camera. The next task is the end effector positioning and orientation which is achieved by image-based approach.

4.2) Camera configurations: There are usually two types of camera configuration found in literature, eye-to-hand, and eye-in-hand. Eye-in-hand configuration can support visual servoing of the hand's end effector towards the target position, while eye-to-Hand can not only provide information about the hand position with respect to the target position but eye-to-Hand can also tell us the relative position of the hand with respect to the platform. Several approaches require the use of eye-to-hand configuration such as Self visual servoing (SVS), will be discussed in the eye-to-hand section below.

Eye-in-hand: In eye-in-hand, the pose of the camera varies with the motion of the robotic arm as well as the multi-rotor. Image-based visual servoing with eye-in-hand camera configuration for aerial manipulation was used by [82]. The study is focused on combined model of aerial manipulator, and a passivity-based adaptive controller is designed which can work on both position and velocity control. This controller is applied to position control so that the aerial manipulator can reach the location of desired objects. Velocity control is used with IBVS to guide the aerial manipulator system.

The papers [51], [20] implement the IBVS using the eye-in-hand camera configuration. The authors use the concept of a ready pose of manipulator with respect to the target object, which enables the manipulator to quickly move around the target object as desired. The goal of the manipulator controller is to maintain that ready pose by using IBVS while considering the host platform's motion as perturbations. Simultaneously the kinematic information obtained from manipulator is used to find the difference 


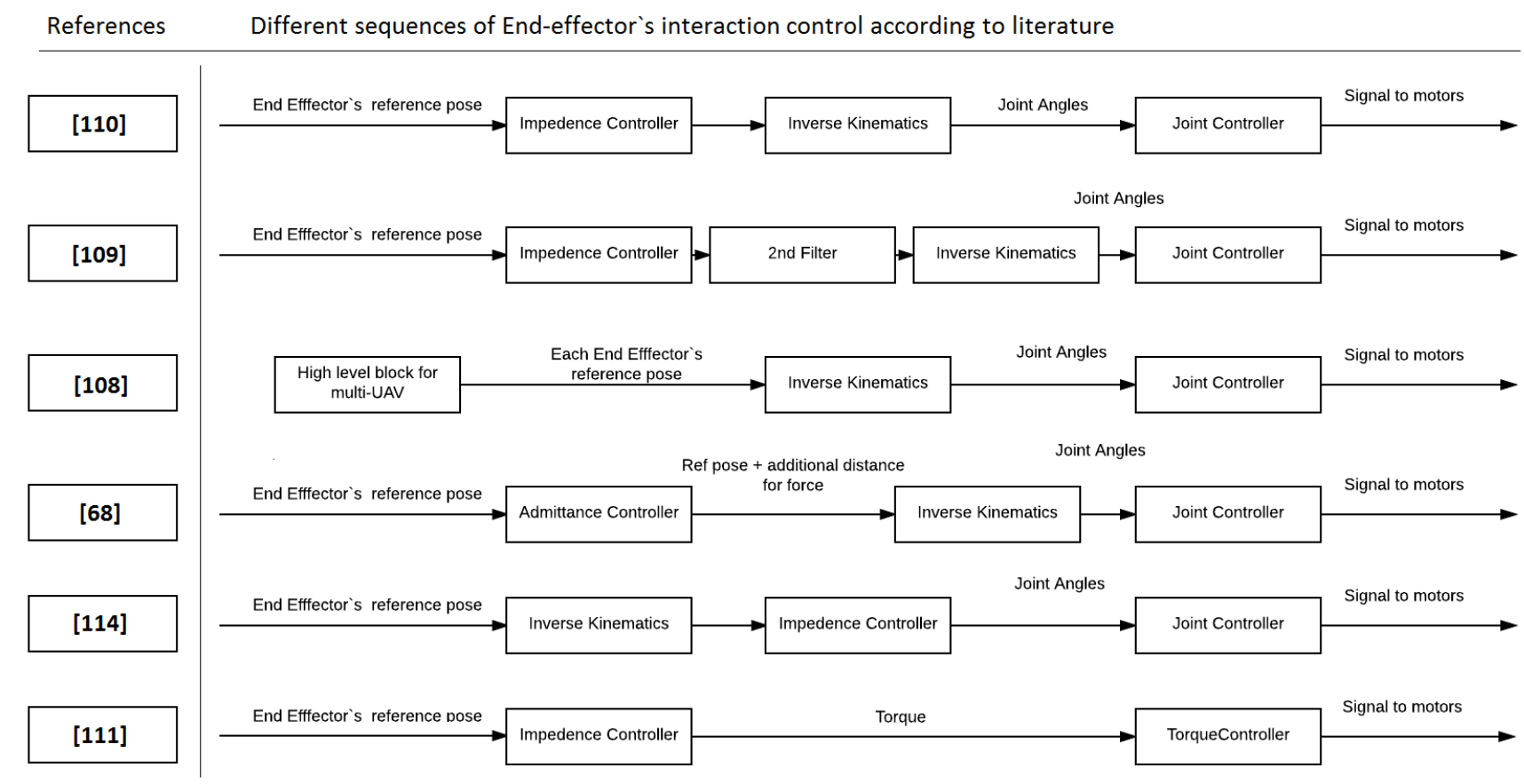

Fig. 7. Different sequences of End-effector's interaction control with environment as proposed in literature

between the ready pose and arm's current pose, and it is used to direct the host platform to move. Eye-in-hand camera configuration was used by [71] for relative positioning of end-effector with respect to the object of interest. Position based visual servoing was used, markers were placed on the object to identify object's relative pose and orientation with respect to the end-effector. In eye-in-hand approach, however, after grasping an object, there is a chance of losing some field of view, therefore after grasping the object, if visual servoing is to be used, an eye-to-hand configuration would be well suited. In [123] a dual arm aerial manipulator is proposed, where one arm is equipped with eye-in-hand camera configuration while the other arm is used to perform the interaction with the environment. The visual information is used to regulate eye-in-hand camera motion with respect to the arm performing the task. The work [123] however needs experimental testing.

Eye-to-hand: Eye-to-hand camera configuration was used by [119] for the placement of the payload on its target location. A control law was developed which calculates an error via IBVS to generate the velocity commands for both UAV and manipulator [119]. The error is based on the image error that is obtained using an eye-to-hand configuration camera. The resulting robotic operation is thus reduced to automatically position an assembly part to the desired location. The placement of payload on target location was also demonstrated by [121] who proposed simultaneous control of UAV and manipulator via velocity commands generated by using image-based visual servoing with the help of an onboard eye-to-hand camera. However, what differs in this study from [119] is the new IBVS technique called self visual servoing (SVS). In this case, the error is the difference in superposition of image features in the carried object and the target's image. Eye-to-hand configuration does not provide the relative position of end-effector with respect to object, but it can be obtained by the knowledge of joint angles or by placing a marker on the end-effector itself. This increases the computational load. There is also a possibility of occlusion of the target object by the manipulator or the end-effector. Eyeto-hand configuration was also used by [122] which was used to get the pose of the object with known markers. In [122] the camera was attached to a $1 \mathrm{DoF}$ joint which allows better tracking of the object.

Latency requirements: The positioning error scales directly with the larger aerial manipulator systems; the end effector position error linearly scales up with the radial length of the manipulator and end effector because of the error in yaw of the aerial platform. It was shown by [35] that latency requirements are crucial for this type of system and time delays in propagation of the signal between perception and actuation components which can significantly affect the overall performance of a visual servoing system. This constraint can be solved by calculating the time delay and to counteract the time delay based on the predicted motion of end effector. The method was verified using experiments.

\section{Multi-UAV aerial manipulation}

Development of aerial platforms for transportation of heavy payload poses restrictions regarding scaling up the UAVs such as multi-rotors as discussed in subsection II$\mathrm{A}$, so it is intuitive to use multiple UAVs to transport a heavy payload. Payloads with complex shapes are also easier 
to carry using multiple UAVs. The orientation control of a larger payload is difficult to manage with a single UAV yaw motion. Multi UAV-collaborative-aerial-manipulator system should be able to deal with the forces imparted by the other UAVs, that are coupled together physically. If an object or payload is held by multiple UAVs, the goal of the control system is to avoid collision amongst UAVs and to ensure that the payload or object is following the desired trajectory, and also the forces acting on the object by the UAVs are bounded [111], [124]. The challenges associated with multiUAV collaborative manipulation also include the difficulty in collision avoidance path planning since the volume of the moving system significantly increases with multi-UAV aerial manipulation [125]. On the other hand, having multiple UAVs provides an opportunity for cooperative localization which can be done via sharing the position estimations of each vehicle with the other [126]. Another challenge is the payload parameter estimation. The inertia and mass of the payload are assumed to be known for the collaborative transport. In [127] a method to estimate inertial parameters of a payload of known geometry is provided. However, in a factory setting or unknown environments geometry of the payload might not be known from before. The control of physically coupled systems can be either via co-ordinated motion or another approach of leader-follower. The following sections V-A and V-B will discuss the leader-follower and co-ordinated motion method in detail.

\section{A. Leader follower approach}

The leader-follower method requires one of the aerial platforms to take the lead, and the follower aerial platform adjusts its motion based on the motion of the leader. Leaderfollower approach for multi-UAV transportation is useful for the cases where the communication between the two aerial platforms is susceptible to communication outages. It does not require a centralized or ground control station to command each aerial platform. However, the challenges associated with the leader-follower approach is the ability to perform complex maneuvers and full attitude control of the payload. Leader-follower approach without communication between two UAVs can be done via two methods. In one of them, follower UAV depends on visual cues from the leader UAV and the gripper and in the other follower UAV applies passive force control to adjust to the motion of the leader. The discussion regarding these two approaches is provided below.

1) Leader follower approach using visual cues: One example of the leader-follower approach is [24] where authors used visual cues for collaboration between leader and follower. One marker is placed on the Leader UAV which allows the follower UAV to maintain altitude and heading alignment with the leader. A pendulum based 1 Degree of freedom manipulator is attached to each UAV with magnetic grippers. Another marker is present on each side of the magnetic gripper's attachment point of the cylindrical payload. The follower keeps itself on top of the payload by tracking the markers on payload using a force controller(PD) based on the distance error, i.e., the offset of the marker from the line below the UAV. In this case only forward or backward motion is allowed, but any lateral movement is not considered.

2) Leader follower approach using passive force control: Another recent example of the leader-follower approach is [128], where the authors use passive force control for the follower UAV while the leader is following the required trajectory. Passive force control was achieved by using an admittance controller that modifies the reference trajectory for the follower by calculating the force acting on the follower. The admittance controller can be tuned by changing the stiffness of the assumed spring mass damper system. More stiffness results in a strict following of the initial trajectory, whereas low stiffness means better compliance to the external forces. The external force acting on the follower is calculated by using the state estimates of the follower and the rotor speeds. There is a possibility of incorrect estimation of force when the follower UAV might perceive any uncertain external force such as wind gusts as the force exerted by the leader. Passive force control seems restricted in orienting the payload in only certain scenarios. Another study by [129] however addresses this problem by choosing a non-zero internal force to induce a required attitude of the payload. This method was extensively simulated by [129] but experimental tests will be required for further assessment of the method. In passive force control, the leader can also not change the direction of motion of the system suddenly or move towards the direction of the follower, while in case of [24], the leader may even move towards the direction of follower and follower may adjust its position. However, these methods are unlikely to be able to perform maneuvers to follow slalom path as it can be performed in co-ordinated motion [126]. The study [128] assumes that the leader and follower altitude are aligned properly, and the method relies on state estimates that were provided using a visual inertial navigation system built for the indoor and outdoor environment.

\section{B. Co-ordinated motion}

Apart from the leader-follower approach, another method of multi-UAV manipulation is via co-ordinated motion. Coordinated motion is defined as a multi-UAV transport strategy, where a centralized station is required for information exchange between the multiple UAVs. In some cases, the centralized station acts in the form of a centralized trajectory generator and controller. Co-ordinated motion method could be in the form of rigid physical coupling between the UAVs and the held object, or it could be in a relatively flexible physical coupling such as holding the payload using cables or multi-link arms. In the case of flexible systems, the scenario can be compared to that of formation control.

1) Formation: Several studies related with the multiUAV collaborative transport of an object showed reliance on maintaining certain configuration in the air, which is a formation approach. These approaches for collaborative transportation are based on a centralized solution, where a centralized system computes a control action for each of 
the UAVs, and it shares the command with them. Most of these studies are based on the absolute position estimation of the UAVs and not the relative positions. Manipulating an object using formation, or a configuration of UAVs in the air is usually achieved by using cable-based systems. The advantage of using cables instead of manipulators or grippers in these systems is that the extension of cable provides more space to the UAVs to spread-out while in flight and thus reduce the chances of collision during formation flight.

An initial study by [31] based on multi-UAV manipulation by co-ordinated motion showed the multi-UAV payload transportation by using three helicopters and cables in outdoor environments. However, a deep analysis was required to ensure static equilibrium of the payload and maintain the stability of the system. Therefore, studies [90], [5], [91] are mainly concerned with maintaining the equilibrium of the payload. In general, the orientation of the payload and the trajectory depends on the position control of the UAVs. The orientation or attitude control of the payload can be useful, in the case of narrow passages, where a particular orientation could be required to pass through.

Specifically, [5] developed spatial configurations for the UAVs that resulted in the static equilibrium of the payload. These configurations were developed considering the constraints on the tension of the cables. These configurations enabled controlling of multiple UAVs while transporting or manipulating the payload. The proposed solution by [5] may result in multiple payload equilibrium solutions. So, non-trivial solutions can be obtained [90] by developing constraints for the UAV spatial configuration that guarantee the existence of a non-trivial payload pose. Another approach regarding the static equilibrium of the payload was presented in [91], where the solution of UAV spatial configuration, was found using inverse kinematics and equilibrium conditions. The authors claimed that if cable tensions are specified, there exists a finite number of solutions and an efficient analytic algorithm based on dialytic elimination was used to find those solutions for the spatial configurations of the UAVs.

A rather different approach was from [130] where modeling conventions of reconfigurable cable-driven parallel robots (RCDPR) was used to derive direct relations between the motion of quadrotors and the motion of payload. This method does not require specification of tensions in cables and uses a tension distribution algorithm to distribute the cable tensions optimally.

The formation approach requires some considerations based on payload type, in all the above cases, the payload is a rigid body with a certain width. There are some cases when the payload is similar to a point mass with a certain width or when it is a deformable linear object (DLO) [131], such as a hose, or cables for building rope-bridges, etc. In such cases, when the DLO is more massive, it would require multiple UAVs to transport it. The weight distribution, in this case, is unequal if all the UAVs have the same altitude, so robot configurations are found using particle swarm optimization that can provide equal-load distribution amongst multiple UAVs carrying the payload [131]. In case, when payload shape is similar to a point mass, i.e., with a smaller width, there are certain implications. During the lift, the UAVs will experience a pull towards each other, and during the transport, this force must be taken into account [132].

The cable-based systems pull the payload, usually upward, but in case, where force application is required a concept presented by [133], [134] could be useful. This concept is multi-UAV collaborative manipulation system based on swarm robotics. The proposed system called, the flying hand is a robotic hand consisting of a swarm of UAVs able to grasp an object where each UAV contributes to the grasping task with a single contact point at the tool-tip. The flying hand, in [133] required the human in the loop, and the UAVs were following the hand gesture of the human. In [134], however, haptic feedback was used. These studies are different because the UAVs collaborate to become a grasping mechanism, while in most cases a gripper with or without an arm manipulator is utilized.

2) Rigid coupling: A payload which is grasped by multiple UAVs using grippers which are rigidly coupled with UAVs does not provide the compliance that is available in case of cable-based multi-UAV and multi-link-manipulator based transportation. The rigid coupling while works for transportation, but it restricts the ability to change pose and orientation of the payload as compared to cable-based systems. The absence of multi-link arms also restricts the ability to move the payload in 3D space while keeping the UAVs in hover. The advantage of having rigid coupling is the availability of constraints in space and time between consecutive position estimates [126] provided by each UAV. If the payload is rigid and the geometry is known, having rigid coupling can, therefore, assist in position estimation in GPS denied environments. In this arrangement co-operative localization is performed where each UAV can benefit from the measurement performed by other UAVs. One study [61] uses grippers attached to the base of the quad-rotor. In this case, a control law is developed based on the payload shape. The proposed control law is based on controlling individual UAVs on their grasping point on the payload. The agents know their grasping position on the payload and the common goal. Due to the knowledge of the grasping position, the orientation and angular estimation of the individual UAV is used to calculate the orientation and angular velocity of the payload. The position and velocity of the center of mass are calculated from the position and velocity of individual UAVs. Each UAV then runs a local hover or velocity controller along with the attitude. In this type of collaborative transport, which relies on centralized co-ordinating station smooth communication is important, any outage in communication can destabilize the system.

\section{Challenges of OUtDoor AERIAL MANiPUlation}

Indoor experiments use motion detectors such as Vicon motion sensors [135] or OptiTrack motion capture systems etc., for accurate tracking of reflective markers indoors. These markers could be placed on objects in order to track them with high precision indoors. Outdoor experiments, 
however, suffer from localization inaccuracies due to the limited GPS accuracy and update rate. Moreover, urban environments with high rise buildings are particularly challenging due to the fact that these environments can block satellite signals and introduce multipath signal challenges that further reduces the accuracy of the location. Localization accuracy is particularly important in aerial manipulation since the UAV is expected to physically interact with the objects or surface. In multi-UAV collaborative aerial manipulation, the localization inaccuracies may result in undesired forces on the jointly carried payload [111].

RTK has been used in certain environments [71] to enhance the quality of the GPS signal and provide a centimeter level localization accuracy. This, however, requires additional RTK base station to be placed in the region where the aerial manipulation is performed. Apart from RTK GPS, techniques such as the Visual Simultaneous Localization and Mapping(VSLAM) [109] has been used in GPS denied environments to handle the outdoor localization challenges. Vision-based navigation [109], visual servoing can be used for outdoor tasks to handle positioning inaccuracies and provide an accurate local relative position suitable for aerial manipulation [92].

Outdoor aerial manipulation should also handle external disturbances introduced by environmental factors such as wind gusts. Disturbance rejection controllers such as [136], [137], have been developed in the past to handle such disturbances. In case of multi-UAV collaborative transport, the outdoor environment can suffer from communication outages, position inaccuracies, and in-sufficient lightning conditions for vision-based methods.

\section{ENERGY MANAGEMENT IN AERIAL MANIPULATION}

The energy consumption in multi-rotors is critical since they are powered by batteries which have limited capacity. The major power consumption results from the motors which are rotating the propellers to generate thrust that keeps the UAV airborne. The electrical energy consumed by the motors depend on the thrust requirements, and also includes the electrical losses and overall propulsion system efficiency. These electrical losses include losses in motors, losses in electronic speed controllers. The resulting flight time of multi-rotors is around 20 to 30 minutes [26]. Attaching a moving manipulator also adds to the energy budget of the aerial platform, regarding added weight and also the energy required by the actuators of the manipulator. So far no study is found which addresses additional energy requirement due to the addition of the moving manipulator or a gripper with actuators. Other causes of energy consumption include the autopilot or any companion computer attached to the aerial platform, sensors such as camera for visual servoing or communication links. The energy constraints in aerial manipulation can be addressed in various stages. One stage is the design stage of the aerial manipulator. The second stage is the energy savings by efficient planning of the operation. The energy savings by design of the aerial manipulator and by efficient planning are discussed below.
Energy savings in design stage can be done in various ways, including reducing the amount of weight carried by the UAV. In some cases, the aerial platform, relies on support from the surroundings, in order to save energy to keep hovering, one example is a novel mechanism proposed by [43] which relies on anchors to keep the platform supported, instead of using the thrust of the propellers. Similarly a gripper on top of the multi-rotor can provide support while the attached manipulator can perform the required operation [138]. In case of [3] the drone supports itself on the wire it is inspecting and hovers only to reach and dock on the wire, or to avoid obstacles during its travel on the wire. If the aerial platform is based on a hybrid design, such as [42] can travel on ground when flight is not necessary to save energy. The aerial platform can perch while doing manipulation to avoid continuously generating the thrust [139]. The energy saving in the second stage is done via efficient planning. In these cases, based on the motor dynamics, minimum energy consumption path can be generated for a single UAV [26]. It is also possible to chose minimum energy consumption path from multiple available paths, generated by a path planner [48] for a single UAV. Another important factor is the mass of the UAV, [140] showed that there is an optimum mass which results in maximum endurance of the UAV flight. In case of specific applications where close contact is required by the UAV, ceiling effect can be used to maximize the flight time [8]. The ceiling effect is similar to ground effects when a UAV is approaching a roof like surface from below it induces additional thrust.

Energy management in multi-UAV collaborative transport is important since power failure in one of the UAVs can fail the whole operation. Although several demonstrations of multi-UAV collaborative transport are present in literature as discussed in section $\mathrm{V}$, none of the papers addressed the energy distribution problem amongst the collaborating UAVs. A study by [141] discussed the need of equal load distribution, in case of transportation of deformable linear objects, the configuration of multiple UAVs which can provide equal-load distribution was estimated using particle swarm optimization. However, in that case, it is assumed that all UAVs are similar and their battery capacities are also the same. Several papers as discussed in section $\mathrm{V}$ are also concerned with changing the pose and orientation of the payload in air [5], [91], while not considering the un-even thrust requirements and hence power distribution. The study by [142] considered the development of a multi-objective control strategy for multi-UAV collaborative transportation where thrust values can be assigned to each vehicle. The mechanism proposed by [142] regulates the thrust requirements; however, this regulation should be done considering the energy availability or the heterogeneity of the UAVs. An optimization algorithm is required to ensure mission completion for heterogeneous collaborative UAVs or homogeneous UAVs with different energy levels. Furthermore, an increase in the volume of the payload nullifies the assumption of point mass and any collaborative transport mechanism should consider the center of gravity of the payload while 
distributing the thrust requirements. Most of the studies for collaborative transport use objects of smaller width and larger length (i.e., higher aspect ratios) whereas in case of lower aspect ratio payload any difference in altitude of the collaborating UAVs will create an uneven thrust requirement thus causing uneven energy distribution. An increase in the depth of the jointly carried payload can also cause shielding of air flow generated by the propellers of the aerial platform which can cause reduction in the thrust generated. This will, in turn, result in more efforts by the propulsion system and hence more energy consumption. The aerodynamic behavior of jointly carried object is an open area of research.

\section{GENERAL DISCUSSION}

In general, the interest of researchers is continuously growing in this field. Below are some generalized observations and research gaps which are identified during the survey.

\section{A. Aerial platform}

1) The novel VTOL aerial platforms as discussed in section II-C which have potential for aerial manipulation are yet to be investigated for addition of an aerial manipulator. For example, there is no study regarding a manipulator attached to a tilt-rotor. Other platforms such as ODAR, or SmQ, can be tested after attaching a manipulator.

2) A stability and efficiency analysis is required for comparing similar scale (capacity and size) helicopters and multi-rotors and attached with similar scale manipulators. This sort of stability analysis is already done for addition of payload mass, but it should be extended to the addition of manipulator as well. This study could answer regarding the favorable platform for attaching a manipulator.

3) The transformable aerial platforms although provide an opportunity for aerial manipulation in terms of variable shape. Having variable shape can allow an aerial platform to navigate through tight spaces. However, this also means that the aerodynamic behavior of the platform would also vary with the transformations. The parasitic drag which becomes significant at higher speeds would also be uncertain, and the problem will become more complex to deal with.

4) The crash-worthiness of aerial manipulation systems needs to be studied and further enhanced.

\section{B. Aerial manipulators and grippers}

1) To the best of our knowledge, soft grippers and manipulators are not yet explored with regards to the aerial manipulation. Soft grippers can provide promising gripping performance and safe interaction with humans. Soft grippers should also be explored for their compliant behavior which can assist in manipulating various types of objects of irregular shapes.

2) A recent study showed that drone delivery could help reduce the greenhouse gases [12]. This, however, cannot be yet said about aerial manipulators. A detailed study is required to perform a life cycle analysis of each of those aerial manipulators specific for each application.

3) Apart from the carbon footprint of the aerial manipulators, it is also necessary to study the financial and feasibility analysis of aerial manipulators vs. the human workers. This is necessary to persuade the end users of aerial manipulation technology to replace human workers from hostile environments.

4) Variable stiffness in compliant grippers and manipulators should be explored.

5) Although haptic feedback for grasping is studied [143]. However, haptic feedback for single and multi-UAV torque applications is needed, since required torque changes from static to dynamic while opening a valve.

6) Aerodynamic effects of a manipulator mounted on UAV can be countered while the manipulator is folded, however, if the manipulator happens to pick an object and performs translational maneuvers, it might still result in loses due to aerodynamic drags.

7) Scaling laws can be developed for aerial manipulators to give a quick idea of how they can be scaled in size, capacity. This can also be supplemented by a stability analysis.

8) There is a "global conditioning index" [88] about the measurement of dexterity, but apart from that, other aspects of aerial manipulation, such as how much manipulation can be performed while being airborne is not discussed. Other performance measures such as financial indicators are not discussed in the literature.

9) A hybrid serial and parallel linked manipulator, could also be investigated, for its behavior as an aerial manipulator. A hybrid manipulator can combine the benefits of both serial and parallel manipulators.

10) In vision-based pick and place of an object, it is possible that the gripper might hinder the view and thus lose track of the Tag/marker. A strategy to deal with such hindrance is needed to ensure smooth pick and place of the payload using vision.

\section{Energy conservation}

1) The additional energy requirements for manipulator motion will reduce the flight time. Although, several papers addressed the control and design of aerial manipulators, yet there is no discussion about increased energy requirements in case of addition of a manipulator to the UAV. A quantification of and scaling of increased energy requirements would help investigate the possibilities of commercialization of aerial manipulation systems.

2) Hardware and algorithm based optimization of the aerial manipulator to reduce energy consumption should be performed.

3) One constraint in commercialized aerial manipulation is the limited energy quota per charge and the time to charge the battery. Energy constraint brings forth the payload capacity limitations, which also restricts 
the weight of the manipulator. A heavy manipulator results in lower flight time.

\section{Multi-UAV aerial manipulation}

1) In multi-UAV collaborative transport, the air flow by the rotors seems to be shielded by the payload. This shielding effect appears to reduce the thrust generated by the rotors. Aerodynamic analysis of such shielding and quantification or scaling of such shielding effect is necessary. There is also a possibility of a multirotor to effect another multi-rotor aerodynamically while jointly transporting a payload. Therefore, a lower bound on the allowable proximity of multi-rotors while picking up and transporting a payload in the air should also be scaled.

2) Multi-UAV transport systems need a mechanism to decide how many multi-rotors would be required to pick up a particular object. This decision does not only depend on the weight of the payload but also on the shape of the payload. Complex shaped objects might result in unusually directed torque on the aerial platforms. Usually in the studies surveyed the problem of object pickup is either ignored or simplified by putting markers. If the shape is complex, then pick up points need to be determined.

3) Multi-UAV collaborative transport should be extended from DLOs to deformable multi-dimensional objects. The research in this area will extend the use of multirotors for applications such as using fishing nets in the oceans.

4) A multi-UAV system for application of torque needs further investigation since with a multi-UAV system we can exert more torque and hence can perform more torsion related operations. A similar system is developed as an SmQ platform, which can apply higher torque using multiple UAVs, but further quantitative analysis of such a system are still needed, and a demonstration of the application of torque is needed.

5) Energy optimization in multi-UAV collaborative transport systems and energy distribution amongst the UAVs during collaborative transport needs exploration.

6) Multi-UAV collaborative transport based on leaderfollower approach, still needs an energy efficient strategy, to transport an object.

7) Multi-UAV collaborative transportation systems are vulnerable to the failures more than single UAV transportation. Mechanisms to deal with a single UAV rotor failure are present which can prevent crashing of the UAV when one or more rotors fail. Similar studies are needed for multi-UAV collaborative systems.

\section{CONCLUSiOn}

Aerial manipulation has the potential for application in areas spreading from agriculture, industry, e-commerce, emergency response, search and rescue. This literature review provided the state-of-the-art in the field of aerial manipulation which could benefit the professionals from diverse fields, to be able to implement aerial manipulation based solutions to their application areas. This literature survey started with the state of the art in platform development for aerial manipulation. Conventional aerial manipulation platforms such as helicopters and multi-rotors were discussed, followed by the survey of development of novel aerial platforms. It was found that platform development is a very active area of research. After the discussion of aerial platforms, the literature review moved forward towards classification of different manipulators and grippers found in literature. It was found that each class of grippers and manipulators exhibits certain attributes which were tabulated and discussed in detail with examples. These attributes include the level of disturbance to the aerial platform caused by different types of manipulators. Researchers have attempted to develop designs which can minimize the disturbance caused by a moving manipulator, but this area of research is still active, and it is expected that many more attempts would be made in recent future in this regard. An important aspect of aerial manipulation is the safe and stable interaction of the aerial manipulator with the environment. These interactions could be for application of force, torque, etc. Researchers have used impedance controllers to achieve safe interaction of endeffector with the environment. Visual servoing is another important means of environment interaction. Researchers have demonstrated the use of visual servoing to move towards the object, pick it up and transport it to the target location.

The recent trend of using multiple UAVs for aerial manipulation was also discussed, with a focus on two basic strategies, leader-follower, and coordinated motion. All these approaches, are susceptible to communication outages, and uncertainties of outdoor conditions, such as insufficient lighting conditions or wind gust, etc. The progress made by researchers cannot be commercialized unless energy constraints are resolved. Currently, energy constraints are the biggest bottleneck in aerial manipulation.

\section{REFERENCES}

[1] Siciliano b. ijars video series: Aerial manipulation. [Online]. Available: DOI: 10.5772/60874

[2] J. J. Acevedo, B. C. Arrue, I. Maza, and A. Ollero, "Cooperative large area surveillance with a team of aerial mobile robots for long endurance missions," Journal of Intelligent \& Robotic Systems, vol. 70, no. 1-4, pp. 329-345, 2013.

[3] W. Chang, G. Yang, J. Yu, Z. Liang, L. Cheng, and C. Zhou, "Development of a power line inspection robot with hybrid operation modes," in IEEE/RSJ International Conference on Intelligent Robots and Systems (IROS). IEEE, 2017, pp. 973-978.

[4] L. Tianyu, L. Yongzhe, Q. Juntong, M. Xiangdong, and H. Jianda, "Modeling and controller design of hydraulic rotorcraft aerial manipulator," in 27th Chinese Control and Decision Conference (CCDC). IEEE, 2015, pp. 5446-5452.

[5] N. Michael, J. Fink, and V. Kumar, "Cooperative manipulation and transportation with aerial robots," Autonomous Robots, vol. 30, no. 1, pp. 73-86, 2011.

[6] J. Cacace, A. Finzi, V. Lippiello, G. Loianno, and D. Sanzone, "Aerial service vehicles for industrial inspection: task decomposition and plan execution," Applied Intelligence, vol. 42, no. 1, pp. 49-62, 2015.

[7] S. Shimahara, S. Leewiwatwong, R. Ladig, and K. Shimonomura, "Aerial torsional manipulation employing multi-rotor flying robot," in 2016 IEEE/RSJ International Conference on Intelligent Robots and Systems (IROS). IEEE, 2016, pp. 1595-1600. 
[8] P. Sánchez-Cuevas, G. Heredia, and A. Ollero, "Multirotor UAS for bridge inspection by contact using the ceiling effect," in International Conference on Unmanned Aircraft Systems (ICUAS). IEEE, 2017, pp. $767-774$

[9] J. R. Kutia, K. A. Stol, and W. Xu, "Aerial manipulator interactions with trees for canopy sampling," IEEE/ASME Transactions on Mechatronics, vol. 23, no. 4, pp. 1740-1749, 2018.

[10] J. M. Gómez-de Gabriel, J. M. Gandarias, F. J. Pérez-Maldonado, F. J. García-Núñcz, E. J. Fernández-García, and A. J. García-Cerezo, "Methods for autonomous wristband placement with a search-andrescue aerial manipulator," in 2018 IEEE/RSJ International Conference on Intelligent Robots and Systems (IROS). IEEE, 2018, pp. 7838-7844.

[11] N. Staub, M. Mohammadi, D. Bicego, D. Prattichizzo, and A. Franchi, "Towards robotic magmas: Multiple aerial-ground manipulator systems," in IEEE International Conference on Robotics and Automation (ICRA). IEEE, 2017, pp. 1307-1312.

[12] J. K. Stolaroff, C. Samaras, E. R. ONeill, A. Lubers, A. S. Mitchell, and D. Ceperley, "Energy use and life cycle greenhouse gas emissions of drones for commercial package delivery," Nature communications, vol. 9 , no. 1, p. 409, 2018

[13] J. Estévez, J. M. Lopez-Guede, and M. Graña, "Quasi-stationary state transportation of a hose with quadrotors," Robotics and Autonomous Systems, vol. 63, pp. 187-194, 2015.

[14] F. Augugliaro, S. Lupashin, M. Hamer, C. Male, M. Hehn, M. W. Mueller, J. S. Willmann, F. Gramazio, M. Kohler, and R. D'Andrea, "The flight assembled architecture installation: Cooperative construction with flying machines," IEEE Control Systems, vol. 34, no. 4, pp. 46-64, 2014.

[15] C. D. Bellicoso, L. R. Buonocore, V. Lippiello, and B. Siciliano, "Design, modeling and control of a 5-DoF light-weight robot arm for aerial manipulation," in 23rd Mediterranean Conference on Control and Automation (MED). IEEE, 2015, pp. 853-858.

[16] K. Kondak, F. Huber, M. Schwarzbach, M. Laiacker, D. Sommer, M. Béjar, and A. Ollero, "Aerial manipulation robot composed of an autonomous helicopter and a 7 degrees of freedom industrial manipulator," in 2014 IEEE International Conference on Robotics and Automation (ICRA). IEEE, 2014, pp. 2107-2112.

[17] C. M. Korpela, T. W. Danko, and P. Y. Oh, "MM-UAV: Mobile manipulating unmanned aerial vehicle," Journal of Intelligent and Robotic Systems: Theory and Applications, vol. 65, no. 1-4, pp. $93-$ 101, 2012.

[18] C. Korpela, M. Orsag, and P. Oh, "Towards valve turning using a dual-arm aerial manipulator," in 2014 IEEE/RSJ International Conference on Intelligent Robots and Systems (IROS 2014). IEEE, 2014, pp. 3411-3416.

[19] L. M. Matteo Fumagalli, Roberto Naldi, Alessandro Macchelli, Francesco Forte, Arvid Q.L. Keemink, Stefano Stramigioli, Raffaella Carloni, "Developing an Aerial Manupulator Prototype," Robotics and Automation, vol. 21, no. 3, pp. 41-50, 2014.

[20] T. W. Danko and P. Y. Oh, "Toward coordinated manipulatorhost visual servoing for mobile manipulating UAVs," in International Conference on Technologies for Practical Robot Applications (TePRA). IEEE, 2014, pp. 1-6.

[21] F. Ruggiero, M. A. Trujillo, R. Cano, H. Ascorbe, A. Viguria, C. Peréz, V. Lippiello, A. Ollero, and B. Siciliano, "A multilayer control for multirotor UAVs equipped with a servo robot arm," in 2015 IEEE international conference on robotics and automation (ICRA). IEEE, 2015, pp. 4014-4020.

[22] B. Theys, G. Dimitriadis, P. Hendrick, and J. De Schutter, "Influence of propeller configuration on propulsion system efficiency of multirotor Unmanned Aerial Vehicles," 2016 International Conference on Unmanned Aircraft Systems, ICUAS 2016, no. June, pp. 195-201, 2016.

[23] W. H. Meier and J. R. OLSON, "Efficient sizing of a cargo rotorcraft," Journal of Aircraft, vol. 25, no. 6, pp. 538-543, 1988

[24] M. Gassner, T. Cieslewski, and D. Scaramuzza, "Dynamic collaboration without communication: Vision-based cable-suspended load transport with two quadrotors," in IEEE International Conference on Robotics and Automation (ICRA). IEEE, 2017, pp. 5196-5202.

[25] D. Mellinger and V. Kumar, "Minimum snap trajectory generation and control for quadrotors," in IEEE International Conference on Robotics and Automation (ICRA). IEEE, 2011, pp. 2520-2525.

[26] F. Morbidi, R. Cano, and D. Lara, "Minimum-energy path generation for a quadrotor uav," in 2016 IEEE International Conference on Robotics and Automation (ICRA). IEEE, 2016, pp. 1492-1498.

[27] S. Park, J. Her, J. Kim, and D. Lee, "Design, modeling and control of omni-directional aerial robot," in International Conference on Intelligent Robots and Systems (IROS). IEEE, 2016, pp. 1570-1575.

[28] M. Ryll, G. Muscio, F. Pierri, E. Cataldi, G. Antonelli, F. Caccavale, and A. Franchi, " $6 \mathrm{D}$ physical interaction with a fully actuated aerial robot," in IEEE International Conference on Robotics and Automation (ICRA). IEEE, 2017, pp. 5190-5195.

[29] T. Kuciński, T. Rybus, K. Seweryn, M. Banaszkiewicz, T. Buratowski, G. Chmaj, J. Grygorczuk, and T. Uhl, "Deployable manipulator technology with application for uavs," in Aerospace Robotics II. Springer, 2015, pp. 93-103.

[30] B. Yang, Y. He, J. Han, and G. Liu, "Rotor-flying manipulator: modeling, analysis, and control," Mathematical Problems in Engineering, vol. 2014, 2014

[31] I. Maza, K. Kondak, M. Bernard, and A. Ollero, "Multi-UAV cooperation and control for load transportation and deployment," Journal of Intelligent and Robotic Systems: Theory and Applications, vol. 57, no. 1-4, pp. 417-449, 2010.

[32] P. E. I. Pounds, D. R. Bersak, and A. M. Dollar, "Stability of smallscale UAV helicopters and quadrotors with added payload mass under PID control," Autonomous Robots, vol. 33, no. 1-2, pp. 129-142, 2012.

[33] K. Kondak, K. Krieger, A. Albu-Schaeffer, M. Schwarzbach, M. Laiacker, I. Maza, A. Rodriguez-Castano, and A. Ollero, "Closed-loop behavior of an autonomous helicopter equipped with a robotic arm for aerial manipulation tasks," International Journal of Advanced Robotic Systems, vol. 10, 2013

[34] K. Kondak, A. Ollero, I. Maza, K. Krieger, A. Albu-Schaeffer, M. Schwarzbach, and M. Laiacker, "Unmanned aerial systems physically interacting with the environment: Load transportation, deployment, and aerial manipulation," in Handbook of Unmanned Aerial Vehicles. Springer, 2015, pp. 2755-2785.

[35] M. Laiacker, F. Huber, and K. Kondak, "High accuracy visual servoing for aerial manipulation using a 7 degrees of freedom industria manipulator," IEEE International Conference on Intelligent Robots and Systems, vol. 2016-November, pp. 1631-1636, 2016.

[36] F. Huber, K. Kondak, K. Krieger, D. Sommer, M. Schwarzbach, M. Laiacker, I. Kossyk, S. Parusel, S. Haddadin, and A. AlbuSchäffer, "First analysis and experiments in aerial manipulation using fully actuated redundant robot arm," in 2013 IEEE/RSJ International Conference on Intelligent Robots and Systems (IROS). IEEE, 2013, pp. 3452-3457.

[37] V. Nayak, C. Papachristos, and K. Alexis, "Design and control of an aerial manipulator for contact-based inspection," arXiv preprint arXiv:1804.03756, 2018.

[38] A. Jimenez-Cano, J. Braga, G. Heredia, and A. Ollero, "Aerial manipulator for structure inspection by contact from the underside," in IEEE/RSJ International Conference on Intelligent Robots and Systems (IROS). IEEE, 2015, pp. 1879-1884.

[39] K. Karydis and V. Kumar, "Energetics in robotic flight at small scales," Interface focus, vol. 7, no. 1, p. 20160088, 2017.

[40] C. Korpela, M. Orsag, T. Danko, B. Kobe, C. McNeil, R. Pisch, and P. Oh, "Flight stability in aerial redundant manipulators," in 2012 IEEE International Conference on Robotics and Automation (ICRA). IEEE, 2012, pp. 3529-3530.

[41] H.-N. Nguyen, S. Park, J. Park, and D. Lee, "A novel robotic platform for aerial manipulation using quadrotors as rotating thrust generators," IEEE Transactions on Robotics, vol. 34, no. 2, pp. 353369,2018

[42] S. Morton and N. Papanikolopoulos, "A small hybrid ground-air vehicle concept," in IEEE/RSJ International Conference on Intelligent Robots and Systems (IROS). IEEE, 2017, pp. 5149-5154.

[43] K. Zhang, P. Chermprayong, T. Alhinai, R. Siddall, and M. Kovac, "Spidermav: Perching and stabilizing micro aerial vehicles with bio-inspired tensile anchoring systems," in IEEE/RSJ International Conference on Intelligent Robots and Systems (IROS). IEEE, 2017, pp. 6849-6854.

[44] M. Zhao, K. Kawasaki, X. Chen, S. Noda, K. Okada, and M. Inaba, "Whole-body aerial manipulation by transformable multirotor with two-dimensional multilinks," in IEEE International Conference on Robotics and Automation (ICRA). IEEE, 2017, pp. 5175-5182.

[45] R. Oung and R. DAndrea, "The distributed flight array: Design, implementation, and analysis of a modular vertical take-off and 
landing vehicle," The International Journal of Robotics Research, vol. 33, no. 3, pp. 375-400, 2014.

[46] A. Nikou, G. C. Gavridis, and K. J. Kyriakopoulos, "Mechanical design, modelling and control of a novel aerial manipulator," in 2015 IEEE International Conference on Robotics and Automation (ICRA). IEEE, 2015, pp. 4698-4703.

[47] Smq platform: A novel robotic platform for aerial manipulation. [Online]. Available: www.youtube.com

[48] J. Economou, G. Kladis, A. Tsourdos, and B. White, "UAV optimum energy assignment using dijkstra's algorithm," in European Control Conference (ECC). IEEE, 2007, pp. 287-292.

[49] M. M. Maia, D. A. Mercado, and F. J. Diez, "Design and implementation of multirotor aerial-underwater vehicles with experimental results," in IEEE/RSJ International Conference on Intelligent Robots and Systems (IROS). IEEE, 2017, pp. 961-966.

[50] C. Korpela, M. Orsag, Y. Jun, P. Brahmbhatt, and P. Oh, "A hardwarein-the-loop test rig for aerial manipulation," in 2013 International Conference on Unmanned Aircraft Systems (ICUAS). IEEE, 2013, pp. 982-987.

[51] T. W. Danko and P. Y. Oh, "Evaluation of visual servoing control of aerial manipulators using test gantry emulation," in 2014 International Conference on Unmanned Aircraft Systems (ICUAS). IEEE, 2014, pp. 821-829.

[52] D. Kim and P. Y. Oh, "Lab automation drones for mobile manipulation in high throughput systems," in 2018 IEEE International Conference on Consumer Electronics (ICCE). IEEE, 2018, pp. 1-5.

[53] G. B. Haberfeld, D. Sun, and N. Hovakimyan, "Stabilization and optimal trajectory generation for a compact aerial manipulation system with a delta-type parallel robot," in 2018 International Conference on Unmanned Aircraft Systems (ICUAS). IEEE, 2018, pp. 1091-1100.

[54] P. E. Pounds and A. M. Dollar, "Aerial grasping from a helicopter UAV platform," in Experimental Robotics. Springer, 2014, pp. 269283.

[55] A. Suarez, G. Heredia, and A. Ollero, "Compliant and lightweight anthropomorphic finger module for aerial manipulation and grasping," in Robot 2015: Second Iberian Robotics Conference. Springer, 2016, pp. 543-555.

[56] A. Suarez, G. Heredia, and R. Ollero, "Lightweight compliant arm with compliant finger for aerial manipulation and inspection," in 2016 IEEE/RSJ International Conference on Intelligent Robots and Systems (IROS). IEEE, 2016, pp. 4449-4454.

[57] S. B. Backus, L. U. Odhner, and A. M. Dollar, "Design of hands for aerial manipulation: Actuator number and routing for grasping and perching," in 2014 IEEE/RSJ International Conference on Intelligent Robots and Systems. IEEE, 2014, pp. 34-40.

[58] S. B. Backus and A. M. Dollar, "A prismatic-revolute-revolute joint hand for grasping from unmanned aerial vehicles and other minimally constrained vehicles," Journal of Mechanisms and Robotics, vol. 10, no. 2, p. 025006, 2018.

[59] L. Kruse and J. Bradley, "A hybrid, actively compliant manipulator/gripper for aerial manipulation with a multicopter," in 2018 IEEE International Symposium on Safety, Security, and Rescue Robotics (SSRR). IEEE, 2018, pp. 1-8.

[60] D. Mellinger, Q. Lindsey, M. Shomin, and V. Kumar, "Design, modeling, estimation and control for aerial grasping and manipulation," in 2011 IEEE/RSJ International Conference on Intelligent Robots and Systems (IROS). IEEE, 2011, pp. 2668-2673.

[61] D. Mellinger, M. Shomin, N. Michael, and V. Kumar, "Cooperative grasping and transport using multiple quadrotors," in Distributed autonomous robotic systems. Springer, 2013, pp. 545-558.

[62] M. Orsag, C. Korpela, M. Pekala, and P. Oh, "Stability control in aerial manipulation," in American Control Conference (ACC). IEEE, 2013, pp. 5581-5586.

[63] A. Gawel, M. Kamel, T. Novkovic, J. Widauer, D. Schindler, B. P. von Altishofen, R. Siegwart, and J. Nieto, "Aerial picking and delivery of magnetic objects with MAVs," in IEEE International Conference on Robotics and Automation (ICRA). IEEE, 2017, pp. 5746-5752.

[64] S. Kim, S. Choi, H. Lee, and H. J. Kim, "Vision-based collaborative lifting using quadrotor UAVs," International Conference on Control, Automation and Systems, no. 2, pp. 1169-1174, 2014.

[65] Nicadrone. [Online]. Available: www.nicadrone.com

[66] N. Toumi, "Decentralized control of multi-agent aerial transportation system," 2017.
[67] U. A. Fiaz, M. Abdelkader, and J. S. Shamma, "An intelligent gripper design for autonomous aerial transport with passive magnetic grasping and dual-impulsive release," in 2018 IEEE/ASME International Conference on Advanced Intelligent Mechatronics (AIM). IEEE, 2018, pp. 1027-1032.

[68] C. C. Kessens, J. Thomas, J. P. Desai, and V. Kumar, "Versatile aerial grasping using self-sealing suction," in 2016 IEEE International Conference on Robotics and Automation (ICRA). IEEE, 2016, pp. $3249-3254$

[69] M. Kovač, J. Germann, C. Hürzeler, R. Y. Siegwart, and D. Floreano, "A perching mechanism for micro aerial vehicles," Journal of MicroNano Mechatronics, vol. 5, no. 3-4, pp. 77-91, 2009.

[70] M. Graule, P. Chirarattananon, S. Fuller, N. Jafferis, K. Ma, M. Spenko, R. Kornbluh, and R. Wood, "Perching and takeoff of a robotic insect on overhangs using switchable electrostatic adhesion," Science, vol. 352, no. 6288, pp. 978-982, 2016.

[71] G. Heredia, A. Jimenez-Cano, I. Sanchez, D. Llorente, V. Vega, J. Braga, J. Acosta, and A. Ollero, "Control of a multirotor outdoor aerial manipulator," in International Conference on Intelligent Robots and Systems (IROS),. IEEE, 2014, pp. 3417-3422.

[72] T. W. Danko and P. Y. Oh, "Design and control of a hyper-redundant manipulator for mobile manipulating unmanned aerial vehicles," Journal of Intelligent and Robotic Systems: Theory and Applications, vol. 73, no. 1-4, pp. 709-723, 2014.

[73] T. Bartelds, A. Capra, S. Hamaza, S. Stramigioli, and M. Fumagalli, "Compliant Aerial Manipulators: Toward a New Generation of Aerial Robotic Workers," IEEE Robotics and Automation Letters, vol. 1, no. 1, pp. 477-483, 2016.

[74] A. Suarez, G. Heredia, and A. Ollero, "Lightweight compliant arm for aerial manipulation," in IEEE/RSJ International Conference on Intelligent Robots and Systems (IROS). IEEE, 2015, pp. 1627-1632.

[75] A. Q. Keemink, M. Fumagalli, S. Stramigioli, and R. Carloni, "Mechanical design of a manipulation system for unmanned aerial vehicles," in International Conference on Robotics and Automation (ICRA). IEEE, 2012, pp. 3147-3152.

[76] J. W. Yeol, D. Toohey, and Y.-W. Hwang, "Design and analysis of a multiple tentacle system for mobile manipulation in micro aerial vehicles," Procedia Computer Science, vol. 105, pp. 7-13, 2017.

[77] B. Yüksel, G. Buondonno, and A. Franchi, "Differential flatness and control of protocentric aerial manipulators with any number of arms and mixed rigid-/elastic-joints," in International Conference on Intelligent Robots and Systems (IROS). IEEE, 2016, pp. 561-566.

[78] J. Thomas, J. Polin, K. Sreenath, and V. Kumar, "Avian-inspired grasping for quadrotor micro UAVs," in ASME 2013 international design engineering technical conferences and computers and information in engineering conference. American Society of Mechanical Engineers, 2013, pp. V06AT07A014-V06AT07A014.

[79] S. Kim, S. Choi, and H. J. Kim, "Aerial manipulation using a quadrotor with a two DoF robotic arm," in 2013 IEEE/RSJ International Conference on Intelligent Robots and Systems (IROS). IEEE, 2013, pp. 4990-4995.

[80] G. Garimella and M. Kobilarov, "Towards model-predictive control for aerial pick-and-place," in 2015 IEEE International Conference on Robotics and Automation (ICRA). IEEE, 2015, pp. 4692-4697.

[81] S. Kim, H. Seo, and H. J. Kim, "Operating an unknown drawer using an aerial manipulator," in 2015 IEEE International Conference on Robotics and Automation (ICRA). IEEE, 2015, pp. 5503-5508.

[82] S. Kim, H. Seo, S. Choi, and H. J. Kim, "Vision-guided aerial manipulation using a multirotor with a robotic arm," IEEE/ASME Transactions on Mechatronics, vol. 21, no. 4, pp. 1912-1923, 2016.

[83] H. Seo, S. Kim, and H. J. Kim, "Aerial grasping of cylindrical object using visual servoing based on stochastic model predictive control," in IEEE International Conference on Robotics and Automation (ICRA). IEEE, 2017, pp. 6362-6368.

[84] A. Suarez, P. R. Soria, G. Heredia, B. C. Arrue, and A. Ollero, "Anthropomorphic, compliant and lightweight dual arm system for aerial manipulation," in IEEE/RSJ International Conference on Intelligent Robots and Systems (IROS). IEEE, 2017, pp. 992-997.

[85] Y. Ohnishi, T. Takaki, T. Aoyama, and I. Ishii, "Development of a 4-joint 3-DOF robotic arm with anti-reaction force mechanism for a multicopter," in IEEE/RSJ International Conference on Intelligent Robots and Systems (IROS). IEEE, 2017, pp. 985-991.

[86] T. Ikeda, S. Yasui, M. Fujihara, K. Ohara, S. Ashizawa, A. Ichikawa, A. Okino, T. Oomichi, and T. Fukuda, "Wall contact by octorotor UAV with one DoF manipulator for bridge inspection," in 
International Conference on Intelligent Robots and Systems (IROS). IEEE, 2017, pp. 5122-5127.

[87] M. Fumagalli, S. Stramigioli, and R. Carloni, "Mechatronic design of a robotic manipulator for unmanned aerial vehicles," in 2016 IEEE/RSJ International Conference on Intelligent Robots and Systems (IROS). IEEE, 2016, pp. 4843-4848.

[88] M. Kamel, K. Alexis, and R. Siegwart, "Design and modeling of dexterous aerial manipulator," in 2016 IEEE/RSJ International Conference on Intelligent Robots and Systems (IROS). IEEE, 2016, pp. $4870-4876$.

[89] J. L. Scholten, M. Fumagalli, S. Stramigioli, and R. Carloni, "Interaction control of an uav endowed with a manipulator," in 2013 IEEE International Conference on Robotics and Automation (ICRA). IEEE, 2013, pp. 4910-4915.

[90] J. Fink, N. Michael, S. Kim, and V. Kumar, "Planning and control for cooperative manipulation and transportation with aerial robots," The International Journal of Robotics Research, vol. 30, no. 3, pp. 324-334, 2011.

[91] Q. Jiang and V. Kumar, "The inverse kinematics of cooperative transport with multiple aerial robots," IEEE Transactions on Robotics, vol. 29, no. 1, pp. 136-145, 2013.

[92] V. Spurnỳ, T. Báča, M. Saska, R. Pěnička, T. Krajník, J. Thomas, D. Thakur, G. Loianno, and V. Kumar, "Cooperative autonomous search, grasping, and delivering in a treasure hunt scenario by a team of unmanned aerial vehicles," Journal of Field Robotics, vol. 36, no. 1, pp. 125-148, 2019.

[93] R. Spica, A. Franchi, G. Oriolo, H. H. Bülthoff, and P. R. Giordano, "Aerial grasping of a moving target with a quadrotor uav," in 2012 IEEE/RSJ International Conference on Intelligent Robots and Systems. IEEE, 2012, pp. 4985-4992.

[94] I. Palunko, A. Faust, P. Cruz, L. Tapia, and R. Fierro, "A reinforcement learning approach towards autonomous suspended load manipulation using aerial robots," in 2013 IEEE International Conference on Robotics and Automation. IEEE, 2013, pp. 4896-4901.

[95] P. Ramon Soria, R. Bevec, B. C. Arrue, A. Ude, and A. Ollero, "Extracting objects for aerial manipulation on UAVs using low cost stereo sensors," Sensors (Switzerland), vol. 16, no. 5, pp. 1-20, 2016.

[96] C. Luo, L. Yu, and P. Ren, "A vision-aided approach to perching a bioinspired unmanned aerial vehicle," IEEE Transactions on Industrial Electronics, vol. 65, no. 5, pp. 3976-3984, 2018.

[97] R. Ladig, S. Leewiwatwong, and K. Shimonomura, "Fpga-based fast response image analysis for orientational control in aerial manipulation tasks," Journal of Signal Processing Systems, pp. 1-11, 2018.

[98] M. Orsag, C. M. Korpela, S. Bogdan, and P. Y. Oh, "Hybrid adaptive control for aerial manipulation," Journal of intelligent \& robotic systems, vol. 73, no. 1-4, pp. 693-707, 2014.

[99] D. Bazylev, A. Kremlev, A. Margun, and K. Zimenko, "Design of control system for a four-rotor UAV equipped with robotic arm," in 7th International Congress on Ultra Modern Telecommunications and Control Systems and Workshops (ICUMT). IEEE, 2015, pp. 144-149.

[100] D. Bazylev, A. Margun, K. Zimenko, and A. Kremlev, "UAV equipped with a robotic manipulator," in 22nd Mediterranean Conference on Control and Automation. IEEE, 2014, pp. 1177-1182.

[101] J. P. S. Buzzato, A. C. Hernandes, M. Becker, and G. A. de Paula Caurin, "Aerial manipulation with six-axis force and torque sensor feedback compensation," in Latin American Robotic Symposium, Brazilian Symposium on Robotics (SBR) and Workshop on Robotics in Education (WRE). IEEE, 2018, pp. 158-163.

[102] M. Orsag, C. Korpela, S. Bogdan, and P. Oh, "Lyapunov based model reference adaptive control for aerial manipulation," in 2013 International Conference on Unmanned Aircraft Systems (ICUAS). IEEE, 2013, pp. 966-973.

[103] S. Di Lucia, G. D. Tipaldi, and W. Burgard, "Attitude stabilization control of an aerial manipulator using a quaternion-based backstepping approach," in European Conference on Mobile Robots (ECMR). IEEE, 2015, pp. 1-6.

[104] J. Acosta, M. Sanchez, and A. Ollero, "Robust control of underactuated aerial manipulators via IDA-PBC," in 53rd Annual Conference on Decision and Control (CDC),. IEEE, 2014, pp. 673-678.

[105] A. Khalifa, M. Fanni, A. Ramadan, and A. Abo-Ismail, "Adaptive intelligent controller design for a new quadrotor manipulation system," in 2013 IEEE International Conference on Systems, Man, and Cybernetics. IEEE, 2013, pp. 1666-1671.
[106] A. Jimenez-Cano, J. Martin, G. Heredia, A. Ollero, and R. Cano, "Control of an aerial robot with multi-link arm for assembly tasks," in International Conference on Robotics and Automation. IEEE, 2013, pp. 4916-4921.

[107] A. Y. Mersha, S. Stramigioli, and R. Carloni, "Exploiting the dynamics of a robotic manipulator for control of UAVs," in International Conference on Robotics and Automation (ICRA). IEEE, 2014, pp. $1741-1746$.

[108] S. Kannan, M. Alma, M. A. Olivares-Mendez, and H. Voos, "Adaptive control of aerial manipulation vehicle," in International Conference on Control System, Computing and Engineering (ICCSCE 2014). IEEE, 2014, pp. 273-278.

[109] V. Ghadiok, J. Goldin, and W. Ren, "On the design and development of attitude stabilization, vision-based navigation, and aerial gripping for a low-cost quadrotor," Autonomous Robots, vol. 33, no. 1-2, pp. 41-68, 2012.

[110] G. Muscio, F. Pierri, M. A. Trujillo, E. Cataldi, G. Giglio, G. Antonelli, F. Caccavale, A. Viguria, S. Chiaverini, and A. Ollero, "Experiments on coordinated motion of aerial robotic manipulators," in 2016 IEEE international conference on robotics and automation (ICRA). IEEE, 2016, pp. 1224-1229.

[111] F. Caccavale, G. Giglio, G. Muscio, and F. Pierri, "Cooperative impedance control for multiple uavs with a robotic arm," in IEEE/RSJ International Conference on Intelligent Robots and Systems (IROS). IEEE, 2015, pp. 2366-2371.

[112] E. Cataldi, G. Muscio, M. A. Trujillo, Y. Rodríguez, F. Pierri, G. Antonelli, F. Caccavale, A. Viguria, S. Chiaverini, and A. Ollero, "Impedance control of an aerial-manipulator: Preliminary results," in 2016 IEEE/RSJ International Conference on Intelligent Robots and Systems (IROS). IEEE, 2016, pp. 3848-3853.

[113] M. Sharifi and H. Sayyaadi, "Nonlinear robust adaptive Cartesian impedance control of UAVs equipped with a robot manipulator," Advanced Robotics, vol. 29, no. 3, pp. 171-186, 2015.

[114] A. Suarez, G. Heredia, and A. Ollero, "Physical-virtual impedance control in ultralightweight and compliant dual-arm aerial manipulators," IEEE Robotics and Automation Letters, vol. 3, no. 3, pp. 2553-2560, 2018.

[115] S. Hamaza, I. Georgilas, and T. Richardson, "An adaptivecompliance manipulator for contact-based aerial applications," in 2018 IEEE/ASME International Conference on Advanced Intelligent Mechatronics (AIM). IEEE, 2018, pp. 730-735.

[116] G. Giglio and F. Pierri, "Selective compliance control for an unmanned aerial vehicle with a robotic arm," in 22nd Mediterranean Conference on Control and Automation. IEEE, 2014, pp. 1190 1195.

[117] H. Sayyaadi and M. Sharifi, "Adaptive impedance control of uavs interacting with environment using a robot manipulator," in 2014 Second RSI ISM International Conference on Robotics and Mechatronics (ICRoM). IEEE, 2014, pp. 636-641.

[118] M. Srikanth, A. Soto, A. Annaswamy, E. Lavretsky, and J.-J. Slotine, "Controlled manipulation with multiple quadrotors," in AIAA Guidance, Navigation, and Control Conference, 2011, pp. 65-47.

[119] R. Mebarki, V. Lippiello, and B. Siciliano, "Image-based control for dynamically cross-coupled aerial manipulation," in International Conference on Intelligent Robots and Systems (IROS). IEEE, 2014, pp. $4827-4833$.

[120] L. R. Buonocore, J. Cacace, and V. Lippiello, "Hybrid visual servoing for aerial grasping with hierarchical task-priority control," in 2015 23rd Mediterranean Conference on Control and Automation (MED). IEEE, 2015, pp. 617-623.

[121] R. ${ }^{\sim}$ Mebarki and V. $\sim$ Lippiello, "Image-based control for aerial manipulation," Asian Journal of Control, vol. 16, no. 3, pp. 646-656, 2014

[122] L. Fang, H. Chen, Y. Lou, Y. Li, and Y. Liu, "Visual grasping for a lightweight aerial manipulator based on nsga-ii and kinematic compensation," in 2018 IEEE International Conference on Robotics and Automation (ICRA). IEEE, 2018, pp. 1-6.

[123] V. Lippiello, G. A. Fontanelli, and F. Ruggiero, "Image-based visualimpedance control of a dual-arm aerial manipulator," IEEE Robotics and Automation Letters, vol. 3, no. 3, pp. 1856-1863, 2018.

[124] K. P. Valavanis and G. J. Vachtsevanos, "Handbook of unmanned aerial vehicles," Handbook of Unmanned Aerial Vehicles, pp. 1-3022, 2015.

[125] H. Lee, H. Kim, and H. J. Kim, "Planning and control for collisionfree cooperative aerial transportation," IEEE Transactions on Automation Science and Engineering, 2016. 
[126] G. Loianno and V. Kumar, "Cooperative transportation using small quadrotors using monocular vision and inertial sensing," IEEE Robotics and Automation Letters, vol. 3, no. 2, pp. 680-687, 2018.

[127] H. Lee and H. J. Kim, "Constraint-based cooperative control of multiple aerial manipulators for handling an unknown payload," IEEE Transactions on Industrial Informatics, vol. 13, no. 6, pp. 2780-2790, 2017.

[128] A. Tagliabue, M. Kamel, S. Verling, R. Siegwart, and J. Nieto, "Collaborative transportation using MAVs via passive force control," in IEEE International Conference on Robotics and Automation (ICRA). IEEE, 2017, pp. 5766-5773.

[129] M. Tognon, C. Gabellieri, L. Pallottino, and A. Franchi, "Aerial comanipulation with cables: The role of internal force for equilibria, stability, and passivity," IEEE Robotics and Automation Letters, vol. 3, no. 3, pp. 2577-2583, 2018.

[130] C. Masone, H. H. Bülthoff, and P. Stegagno, "Cooperative transportation of a payload using quadrotors: A reconfigurable cabledriven parallel robot," in 2016 IEEE/RSJ International Conference on Intelligent Robots and Systems (IROS). IEEE, 2016, pp. 16231630.

[131] J. E. Sanz, "Quadrotor Team Modeling and Control for DLO Transportation," 2016.

[132] I. H. B. Pizetta, A. S. Brandao, and M. Sarcinelli-Filho, "Cooperative quadrotors carrying a suspended load," 2016 International Conference on Unmanned Aircraft Systems, ICUAS 2016, pp. 1049-1055, 2016

[133] G. Gioioso, A. Franchi, G. Salvietti, S. Scheggi, and D. Prattichizzo, "The flying hand: A formation of uavs for cooperative aerial telemanipulation," in 2014 IEEE International Conference on Robotics and Automation (ICRA). IEEE, 2014, pp. 4335-4341.

[134] M. Mohammadi, A. Franchi, D. Barcelli, and D. Prattichizzo, "Cooperative aerial tele-manipulation with haptic feedback," in 2016 IEEE/RSJ International Conference on Intelligent Robots and Systems (IROS). IEEE, 2016, pp. 5092-5098.

[135] D. Montufar, F. Munoz, E. Espinoza, O. Garcia, and S. Salazar, "Multi-uav testbed for aerial manipulation applications," in 2014
International Conference on Unmanned Aircraft Systems (ICUAS). IEEE, 2014, pp. 830-835.

[136] J. Bannwarth, Z. Chen, K. Stol, and B. MacDonald, "Disturbance accomodation control for wind rejection of a quadcopter," in 2016 International Conference on Unmanned Aircraft Systems (ICUAS). IEEE, 2016, pp. 695-701.

[137] Y. Zhang, Z. Chen, X. Zhang, Q. Sun, and M. Sun, "A novel control scheme for quadrotor uav based upon active disturbance rejection control," Aerospace Science and Technology, vol. 79, pp. 601-609, 2018.

[138] H. Paul, K. Ono, R. Ladig, and K. Shimonomura, "A multirotor platform employing a three-axis vertical articulated robotic arm for aerial manipulation tasks," in 2018 IEEE/ASME International Conference on Advanced Intelligent Mechatronics (AIM). IEEE, 2018, pp. 478-485.

[139] C. E. Doyle, J. J. Bird, T. A. Isom, C. J. Johnson, J. C. Kallman, J. A Simpson, R. J. King, J. J. Abbott, and M. A. Minor, "Avian-inspired passive perching mechanism for robotic rotorcraft," in IEEE/RS International Conference on Intelligent Robots and Systems (IROS). IEEE, 2011, pp. 4975-4980.

[140] A. Abdilla, A. Richards, and S. Burrow, "Power and endurance modelling of battery-powered rotorcraft," in 2015 IEEE/RSJ International Conference on Intelligent Robots and Systems (IROS). IEEE, 2015, pp. 675-680.

[141] J. Estevez and M. Graña, "Improved Control of DLO Transportation by a Team of Quadrotors," in International Work-Conference on the Interplay Between Natural and Artificial Computation. Springer, 2017, pp. 117-126.

[142] J. Gimenez, D. C. Gandolfo, L. R. Salinas, C. Rosales, and R. Carelli, "Multi-objective control for cooperative payload transport with rotorcraft UAVs," ISA transactions, 2018.

[143] Y. Wu, J. Song, J. Sun, F. Zhu, and H. Chen, “Aerial Grasping Based on VR Perception and Haptic Control," in 2018 IEEE International Conference on Real-time Computing and Robotics (RCAR). IEEE, 2018, pp. 556-562. 